\title{
Universal Approximation of a Class of Interval Type-2 Fuzzy Neural Networks in Nonlinear Identification
}

\author{
Oscar Castillo, ${ }^{1}$ Juan R. Castro, ${ }^{2}$ Patricia Melin, ${ }^{1}$ and Antonio Rodriguez-Diaz ${ }^{2}$ \\ ${ }^{1}$ Tijuana Institute of Technology, 22379 Tijuana, BCN, Mexico \\ ${ }^{2}$ Baja California Autonomous University (UABC), 22379 Tijuana, BCN, Mexico
}

Correspondence should be addressed to Oscar Castillo; ocastillo@hafsamx.org

Received 15 January 2013; Revised 20 June 2013; Accepted 20 June 2013

Academic Editor: F. Herrera

Copyright (c) 2013 Oscar Castillo et al. This is an open access article distributed under the Creative Commons Attribution License, which permits unrestricted use, distribution, and reproduction in any medium, provided the original work is properly cited.

Neural networks (NNs), type-1 fuzzy logic systems (T1FLSs), and interval type-2 fuzzy logic systems (IT2FLSs) have been shown to be universal approximators, which means that they can approximate any nonlinear continuous function. Recent research shows that embedding an IT2FLS on an NN can be very effective for a wide number of nonlinear complex systems, especially when handling imperfect or incomplete information. In this paper we show, based on the Stone-Weierstrass theorem, that an interval type-2 fuzzy neural network (IT2FNN) is a universal approximator, which uses a set of rules and interval type-2 membership functions (IT2MFs) for this purpose. Simulation results of nonlinear function identification using the IT2FNN for one and three variables and for the Mackey-Glass chaotic time series prediction are presented to illustrate the concept of universal approximation.

\section{Introduction}

Several authors have contributed to universal approximation results. An overview can be found in [1-8]; further references to prime contributors in function approximations by neural networks are in [4, 9-12] and type-2 fuzzy logic modeling in [13-23]. It has been shown that a three-layer NN can approximate any real continuous function [24]. The same has been shown for a T1FLS $[1,25]$ using the Stone-Weierstrass theorem [3]. A similar analysis was made by Kosko [2, 9] using the concept of fuzzy regions. In $[3,26]$ Buckley shows that, with a Sugeno model [27], a T1FLS can be built with the ability to approximate any nonlinear continuous function. Also, combining the neural and fuzzy logic paradigms [28, 29], an effective tool can be created for approximating any nonlinear function [4]. In this sense, an expert can use a type-1 fuzzy neural network (T1FNN) $[10-12,30]$ or IT2FNN systems and find interpretable solutions [15-17, 31-34]. In general, Takagi-Sugeno-Kang (TSK) T1FLSs are able to approximate by the use of polynomial consequent rules $[7,27]$. This paper uses the Levenberg-Marquardt backpropagation learning algorithm for adapting antecedent and consequent parameters for an adaptive IT2FNN, since its efficiency and soundness characteristics make them fit for these optimizing problems. An Adaptive IT2FNN is used as a universal approximator of any nonlinear functions. A set $\Psi$ of IT2FNNs is universal if and only if (iff), given any process $\Omega$, there is a system $\Phi \in \Psi$ such that the difference between the output from IT2FNN and output from $\Omega$ is less than a given $\varepsilon$.

In this paper the main contribution is the proposed IT2FNNs architectures, which are shown to be universal approximators and are illustrated with several benchmark problems to verify their applicability for real world problems.

\section{Interval Type-2 Fuzzy Neural Networks}

An IT2FNN [15, 31, 35] combines a TSK interval type-2 fuzzy inference system (TSKIT2FIS) $[13,14,33,34]$ with an adaptive $\mathrm{NN}$ in order to take advantage of both models best characteristics. In general, when representing IT2FNN graphically, rectangles are used to represent adaptive nodes and circles to represent nonadaptive nodes. Output values of pair nodes (green color) and odd nodes (blue color) represent uncertainty intervals (Figures 1-4). In this kind of interval 


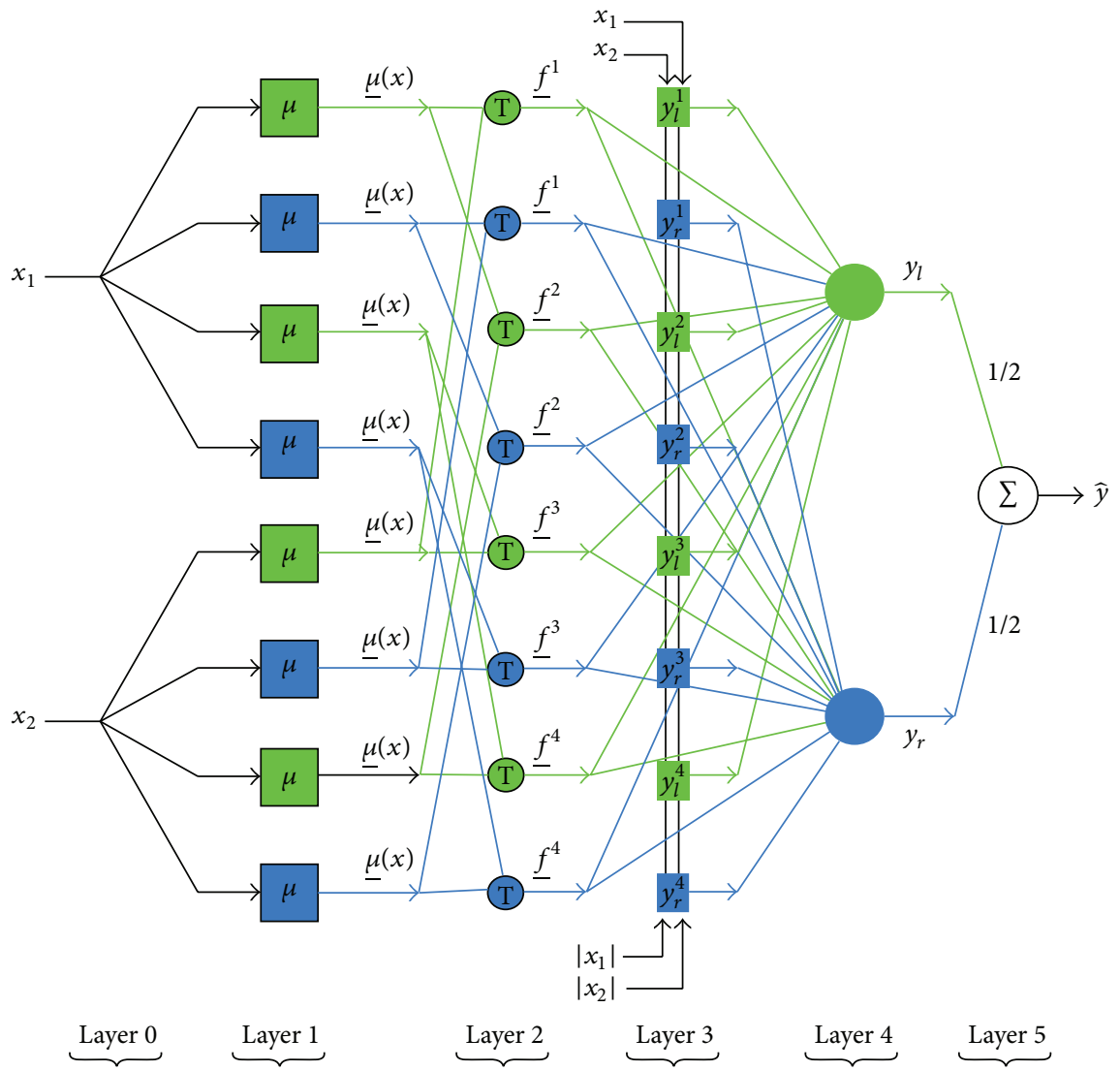

FIGURE 1: IT2FNN-1 architecture.

type-2 neurofuzzy adaptive networks, nodes represent processing units called neurons, which can be classified into crisp and fuzzy neurons.

The IT2FNN-1 architecture has 5 layers (Figure 1) [35] and consists of adaptive nodes with equivalent function to lower-upper membership in fuzzification layer (layer 1). Nonadaptive nodes in rules layer (layer 2) interconnect with the fuzzification layer (layer 1) in order to generate TSK IT2FIS rules antecedents. Adaptive nodes in the consequent layer (layer 3) are connected to the input layer (layer 0) to generate rules consequents. Nonadaptive nodes in typereduction layer (layer 4) evaluate left-right values with the Karnik and Mendel (KM) $[13,14]$ algorithm. The nonadaptive nodes in the defuzzification layer (layer 5) average left-right values.

The IT2FNN-3 architecture has 8 layers (Figure 2) [35] and uses IT2FN for fuzzifying the inputs (layers 1-2). Nonadaptive nodes in rules layer (layer 3 ) interconnect with lower-upper linguistic values layer (layer 2) to generate TSK IT2FIS rules antecedents. Adaptive nodes in layer 4 adapt leftright firing strength, biasing rules lower-upper trigger forces with synaptic weights between layers 3 and 4. Layer 5's nonadaptive nodes normalize rules lower-upper firing strength. Nonadaptive nodes I consequent layer (layer 6) interconnect with input layer (layer 0) to generate rules consequents. Nonadaptive nodes in type-reduction layer (layer 7) evaluate left-right values adding lower-upper product of lower-upper triggering forces normalized by rules consequent left-right values. Node in defuzzification layer is adaptive and its output $\hat{y}$ is defined as biased average of left-right values and parameter $\gamma$. Parameter $\gamma(0.5$ by default $)$ adjusts uncertainty interval defined by left-right values $\left[\hat{y}_{l}, \widehat{y}_{r}\right]$.

Architectures IT2FNN-0 and IT2FNN-2, which will be shown in Sections 3.2 and 3.3, respectively, as universal approximators, are described with more details in Section 2.1.

2.1. IT2FNN-O Architecture. An IT2FNN-0 is a seven-layer IT2FNN, which integrates a first order TSKIT2FIS (interval type-2 fuzzy antecedents and real consequents) with an adaptive NN. The IT2FNN-0 (Figure 3) layers are described as follows.

Layer 0. Inputs

$$
o_{i}^{0}=x_{i}, \quad i=1, \ldots, n
$$

Layer 1. Adaptive type-1 fuzzy neuron (T1FN)

$$
\operatorname{net}_{k}^{1}=w_{k, i}^{1,0} x_{i}+b_{k}^{1} \quad \forall i=1, \ldots, n, k=1, \ldots, \vartheta,
$$

$o_{k}^{1}=\mu\left(\right.$ net $\left._{k}^{1}\right)$, where the transfer function $\mu$ is a membership function, net $_{k}^{1}$ is the weighted sum of inputs $\left(x_{i}\right)$ and 


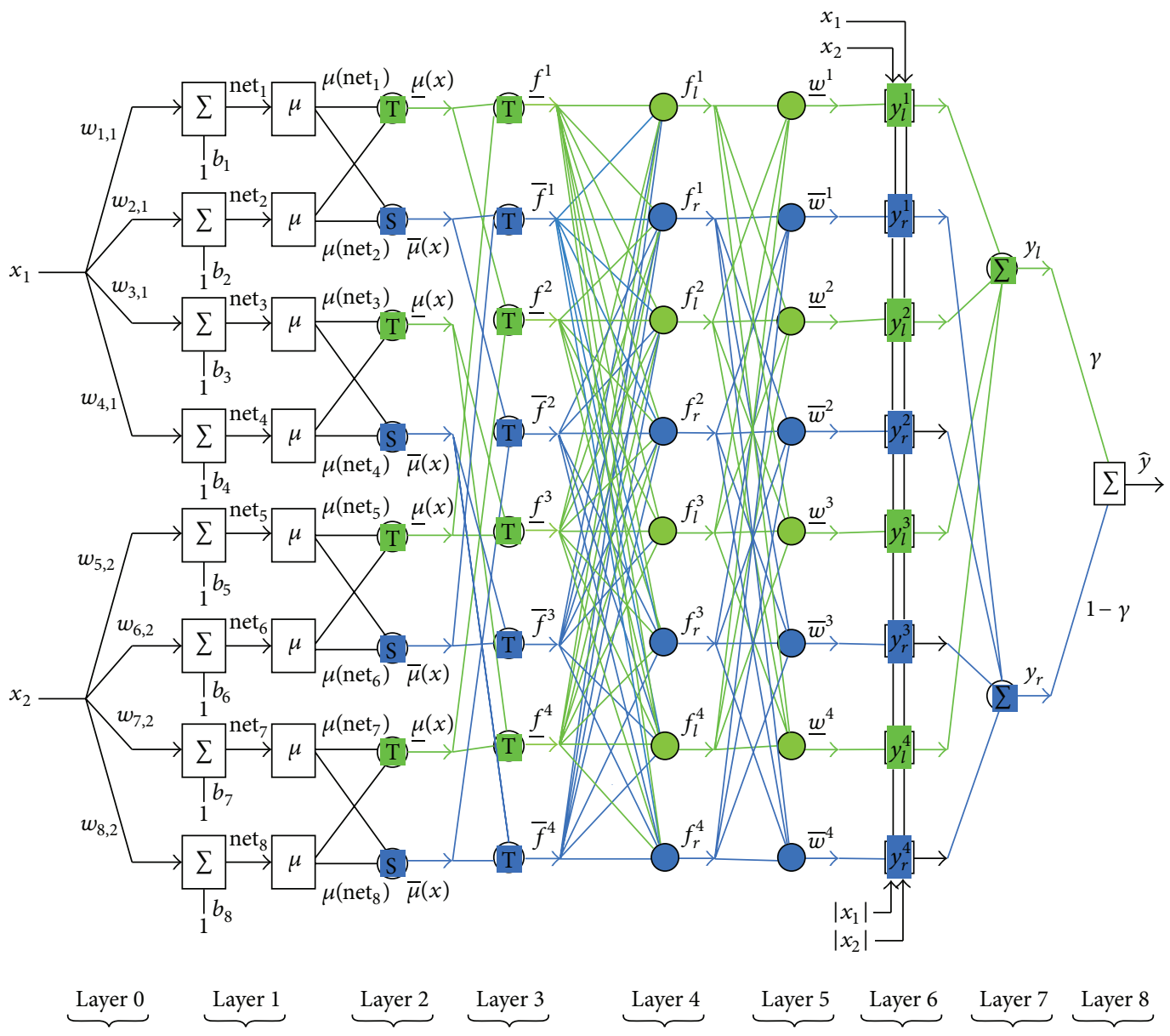

FIGURE 2: IT2FNN-3 architecture.

the synaptic weights $\left(w_{k, i}^{1,0}\right)$, and $b_{k}^{1}$ is the threshold for each neuron.

Layer 2. Nonadaptive T1FN. This layer contains T-norm and S-norm fuzzy nodes

$$
o_{2 k-1}^{2}=o_{2 k-1}^{1} \cdot o_{2 k}^{1} \quad \forall k=1, \ldots, \vartheta, \text { T-norm fuzzy node, }
$$

where $\vartheta$ is the number of nodes in layers 1 and 2

$$
o_{2 k}^{2}=o_{2 k-1}^{1}+o_{2 k}^{1}-o_{2 k-1}^{2}, \quad \text { S-norm fuzzy node, }
$$

$\tau=\ell_{k, i}$ for all $k=1, \ldots, M$, and $i=1, \ldots, n$, where $\ell_{k, i}$ is the table of indices of the antecedents of the rules $\pi=\sum_{j=1}^{i-1} v_{j}+|\tau|$, where $\pi$ is a vector of indices for each node of layer 2

if $\tau>0$

$$
\underline{\mu}_{i}^{k}=o_{i_{l}(\pi)}^{2}, \quad \bar{\mu}_{i}^{k}=o_{i_{u}(\pi)}^{2}
$$

else

$$
\underline{\mu}_{k, i}=\text { null, } \quad \bar{\mu}_{k, i}=\text { null }
$$

end, where $\mu_{i}^{k}, \bar{\mu}_{i}^{k}$ are lower and upper membership function values, respectively. $i_{l}(\pi)$ and $i_{u}(\pi)$ are vectors with even and odd indices of the nodes of layer 2 .

Layer 3. Lower-upper firing strength $\left(w^{k}, \bar{w}^{k}\right)$. Having nonadaptive nodes for generating lower-upper firing strength of TSK IT2FIS rules (7),

$$
\begin{gathered}
o_{2 k-1}^{3}=\underline{w}^{k}, \quad o_{2 k}^{3}=\bar{w}^{k}, \\
\underline{w}^{k}=\prod_{i=1}^{n} \underline{\mu}_{\widetilde{F}_{i}^{k}}(x), \\
\bar{w}^{k}=\prod_{i=1}^{n} \bar{\mu}_{\widetilde{F}_{i}^{k}}(x),
\end{gathered}
$$

where $\mu_{\widetilde{F}_{i}^{k}(x)} \in\left[\underline{\mu}_{\widetilde{F}_{i}^{k}}(x), \bar{\mu}_{\widetilde{F}_{i}^{k}}(x)\right]$ is the Gaussian interval type-2 membership function, igaussmtype $2\left(x,\left[\sigma_{i}^{k}, m_{i}^{k}\right.\right.$, $\left.\left.{ }^{2} m_{i}^{k}\right]\right)$, defined by

$$
{ }^{1} \mu_{\widetilde{F}_{i}^{k}}\left(x_{i},\left[\sigma_{i}^{k}, m_{i}^{k}\right]\right)=\exp \left[-\frac{1}{2}\left(\frac{x_{i}-{ }^{1} m_{i}^{k}}{\sigma_{i}^{k}}\right)^{2}\right],
$$




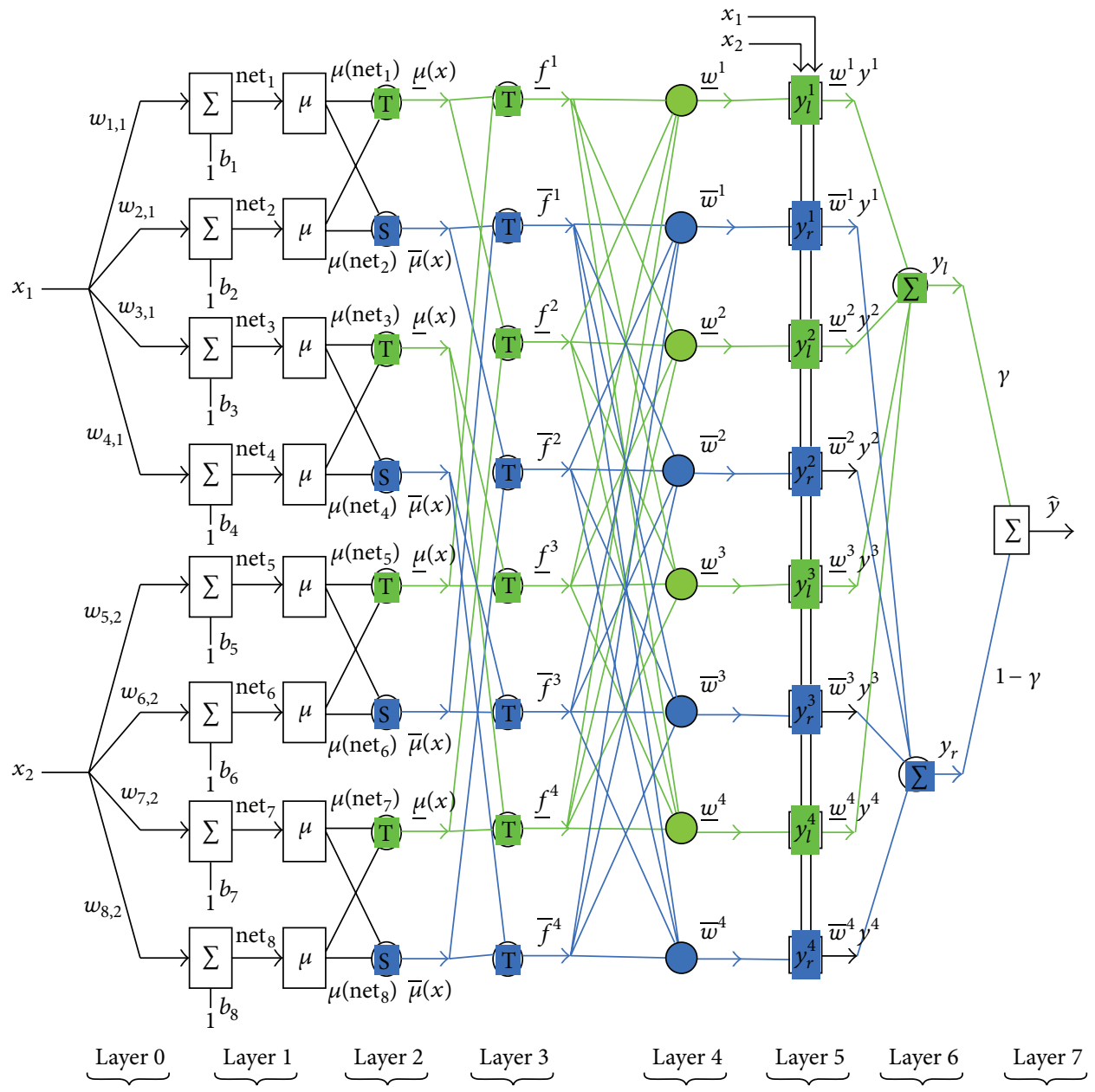

FIGURE 3: IT2FNN-0 architecture.

$$
\begin{gathered}
{ }^{2} \mu_{\widetilde{F}_{i}^{k}}\left(x_{i},\left[\sigma_{i}^{k},{ }^{2} m_{i}^{k}\right]\right)=\exp \left[-\frac{1}{2}\left(\frac{x_{i}-{ }^{2} m_{i}^{k}}{\sigma_{i}^{k}}\right)^{2}\right], \\
\underline{\mu}_{F_{i}^{k}}(x)= \begin{cases}{ }^{2} \mu_{\widetilde{F}_{i}^{k}}\left(x_{i},\left[\sigma_{i}^{k}, m_{i}^{k}\right]\right), & x_{i} \leq m_{i}^{k}, \\
1 \mu_{\widetilde{F}_{i}^{k}}\left(x_{i},\left[\sigma_{i}^{k}, m_{i}^{k}\right]\right), & x_{i}>^{2} m_{i}^{k},\end{cases} \\
\bar{\mu}_{F_{i}^{k}}(x)= \begin{cases}{ }^{1} \mu_{\widetilde{F}_{i}^{k}}\left(x_{i},\left[\sigma_{i}^{k}, m_{i}^{k}\right]\right), & x_{i}<{ }^{1} m_{i}^{k}, \\
1, & { }^{1} m_{i}^{k} \leq x_{i} \leq m_{i}^{k}, \\
2 \mu_{\widetilde{F}_{i}^{k}}\left(x_{i},\left[\sigma_{i}^{k}, m_{i}^{k}\right]\right), & x_{i}>{ }^{2} m_{i}^{k} .\end{cases}
\end{gathered}
$$

Layer 4. Lower-upper firing strength rule normalization $\left(\underline{\phi}^{k}, \bar{\phi}^{k}\right)$. Nodes in this layer are nonadaptive and the output is defined as the ratio between the $k$ th lower-upper firing strength rule $\left(\underline{w}^{k}, \bar{w}^{k}\right)$ and the sum of lower-upper firing strength of all rules (13) and (14):

$$
o_{2 k-1}^{4}=\underline{\phi}^{k}, \quad o_{2 k}^{4}=\bar{\phi}^{k}
$$

If we view $\phi^{k}, \bar{\phi}^{k}$ as fuzzy basis functions (FBF) (32) and (33) and $y^{k}(x)$ as linear function (16), then $\widehat{y}(x)$ can be viewed as a linear combination of the basis functions (20) and (21):

$$
\underline{\phi}^{k}=\frac{\underline{w}^{k}}{D_{l}}, \quad k=1, \ldots, M,
$$

where $D_{l}=\sum_{k=1}^{M} \underline{w}^{k}$,

$$
\bar{\phi}^{k}=\frac{\bar{w}^{k}}{D_{r}}, \quad k=1, \ldots, M,
$$

where $D_{r}=\sum_{k=1}^{M} \bar{w}^{k}$.

Layer 5. Rule consequents. Each node is adaptive and its parameters are $\left\{c_{i}^{k}, c_{0}^{k}\right\}$. The node's output corresponds to partial output of $k$ th rule $y^{k}(16)$ :

$$
\begin{array}{cc}
o_{2 k=1}^{5}=y^{k}, & o_{2 k}^{5}=y^{k} \\
y^{k}=\sum_{i=1}^{n} c_{i}^{k} x_{i}+c_{0}^{k} ; & k=1, \ldots, M .
\end{array}
$$




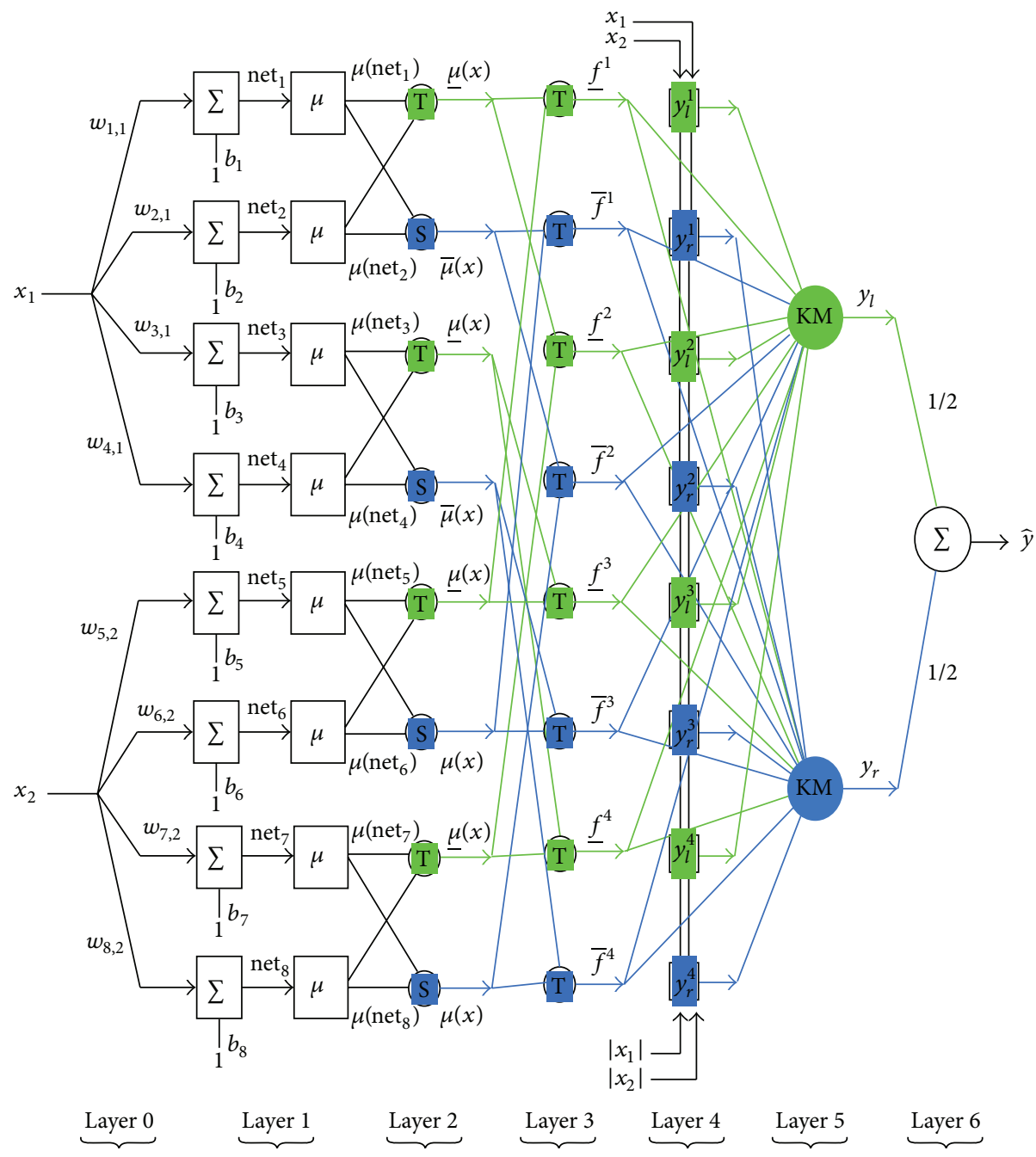

FIGURE 4: IT2FNN-2 architecture.

Layer 6. Estimating left-right interval values $\left[\hat{y}_{l}, \hat{y}_{r}\right]$ (18), nodes are nonadaptive with outputs $\widehat{y}_{l}, \widehat{y}_{r}$. Layer 6 output is defined by

$$
o_{1}^{6}=\widehat{y}_{l}(x), \quad o_{2}^{6}=\widehat{y}_{r}(x)
$$

where

$$
\begin{aligned}
& \widehat{y}_{l}(x)=\sum_{k=1}^{M} \underline{\phi}^{k} y^{k}, \\
& \widehat{y}_{r}(x)=\sum_{k=1}^{M} \bar{\phi}^{k} y^{k} .
\end{aligned}
$$

Layer 7. Defuzzification. This layer's node is adaptive, where the output $\hat{y},(20)$ and (21), is defined as weighted average of left-right values and parameter $\gamma$. Parameter $\gamma$ (default value 0.5 ) adjusts the uncertainty interval defined by left-right values $\left[\widehat{y}_{l}, \widehat{y}_{r}\right]$ :

$$
o_{1}^{7}=\widehat{y}(x)
$$

where

$$
\begin{gathered}
\widehat{y}(x)=\gamma \widehat{y}_{l}(x)+(1-\gamma) \widehat{y}_{r}(x), \\
\widehat{y}(x)=\sum_{k=1}^{M}\left[\gamma \underline{\phi}^{k}(x)+(1-\gamma) \bar{\phi}^{k}(x)\right] y^{k}(x) .
\end{gathered}
$$

2.2. IT2FNN-2 Architecture. An IT2FNN-2 [31] is a sixlayer IT2FNN, which integrates a first order TSKIT2FIS (interval type-2 fuzzy antecedents and interval type-1 fuzzy consequents), with an adaptive NN. The IT2FNN-2 (Figure 4) layers are described in a similar way to the previous architectures. 


\section{IT2FNN as a Universal Approximator}

Based on the description of the interval type-2 fuzzy neural networks, it is possible to prove that under certain conditions, the resulting IT2FIS has unlimited approximation power to match any nonlinear functions on a compact set $[36,37]$ using the Stone-Weierstrass theorem $[5,6,10,30]$.

\subsection{Stone-Weierstrass Theorem}

Theorem 1 (Stone-Weierstrass theorem). Let $Z$ be a set of real continuous functions on a compact set $U$. If (1) $Z$ is an algebra, that is, the set $Z$ is closed under addition, multiplication, and scalar multiplication, (2) $Z$ separates points on $U$, that is, for every $\mathbf{x}, \mathbf{y} \in U, \mathbf{x} \neq \mathbf{y}$, there exists $f \in Z$ such that $f(\mathbf{x}) \neq f(\mathbf{y})$, and (3) $Z$ vanishes at no point of $U$, that is, for each $x \in U$ there exists $f \in Z$ such that $f(\mathbf{x}) \neq 0$, then the uniform closure of $Z$ consists of all real continuous functions on $U$; that is, $\left(Z, d_{\infty}\right)$ is dense in $\left(C[U], d_{\infty}\right)$ [36-38].

Theorem 2 (universal approximation theorem). For any given real continuous function $g(u)$ on the compact set $U \subset R^{n}$ and arbitrary $\varepsilon>0$, there exists $f \in Y$ such that $\sup _{x \in U}(\mid g(x)-$ $f(x) \mid)<\varepsilon$.

3.2. Applying Stone-Weierstrass Theorem to the IT2FNN-0 Architecture. In the IT2FNN-0, the domain on which we operate is almost always compact. It is a standard result in real analysis that every closed and bounded set in $\mathfrak{R}^{n}$ is compact. Now we shall apply the Stone-Weierstrass theorem to show the representational power of IT2FNN with simplified fuzzy if-then rules. We now consider a subset of the IT2FNN-0 on Figure 5. The set of IT2FNN-0 with singleton fuzzifier, product inference, center of sets type reduction, and Gaussian interval type- 2 membership function consists of all FBF expansion functions of the form (38), (40). $f: U \subset$ $R^{n} \rightarrow R, x=\left(x_{1}, x_{2}, \ldots, x_{n}\right) \in U ; \mu_{\widetilde{F}_{i}^{k}(x)} \in\left[\underline{\mu}_{\widetilde{F}_{i}^{k}}(x)\right.$, $\left.\bar{\mu}_{\widetilde{F}_{i}^{k}}(x)\right]$ is the Gaussian interval type-2 membership function, igaussmtype $2\left(x,\left[\sigma_{i}^{k},{ }^{1} m_{i}^{k}, m_{i}^{k}\right]\right)$, defined by (27) and (31). If we view $\phi^{k}(x), \bar{\phi}^{k}(x)$ as fuzzy basis functions (32) and (33) and $y^{k}(x)$ are linear functions (34), then $\hat{y}(x)$ of (38) and (40) can be viewed as a linear combination of the fuzzy basis functions, and then the IT2FNN-0 system is equivalent to an FBF expansion. Let $Y$ be the set of all the FBF expansions (38) and (40) with $\phi^{k}(x), \bar{\phi}^{k}(x)$ given by (13) and (38) and let $d_{\infty}\left(f_{1}, f_{2}\right)=\sup _{x \in U}\left(\left|f_{1}(x)-f_{2}(x)\right|\right)$ be the supmetric; then, $\left(Y, d_{\infty}\right)$ is a metric space [38]. We use the following StoneWeierstrass theorem to prove our result.

Suppose we have two IT2FNN-0s $f_{1}, f_{2} \in Y$; the output of each system can be expressed as

$$
f_{1}(x)=\alpha \widehat{y}_{l}^{1}(x)+(1-\alpha) \widehat{y}_{r}^{1}(x),
$$

where

$$
\begin{aligned}
& \widehat{y}_{l}^{1}(x)=\sum_{k=1}^{M} \underline{\phi}_{1}^{k}(x) z_{1}^{k}(x)=\frac{\sum_{k=1}^{M_{1}} \underline{w}_{1}^{k}(x) z_{1}^{k}(x)}{D_{l}^{1}}, \\
& \hat{y}_{r}^{1}(x)=\sum_{k=1}^{M_{1}} \underline{\phi}_{1}^{k}(x) z_{1}^{k}(x)=\frac{\sum_{k=1}^{M_{1}} \bar{w}_{1}^{k}(x) z_{1}^{k}(x)}{D_{r}^{1}},
\end{aligned}
$$

where

$$
\begin{gathered}
D_{l}^{1}=\sum_{k=1}^{M_{1}} \prod_{i=1}^{n} \underline{\mu}_{1} \widetilde{F}_{i}^{k}(x), \quad D_{r}^{1}=\sum_{k_{1}=1}^{M_{1}} \prod_{i=1}^{n} \bar{\mu}_{1} \widetilde{F}_{i}^{k}(x), \\
\underline{w}_{1}^{k}=\prod_{i=1}^{n} \underline{\mu}_{1} \widetilde{F}_{i}^{k}(x), \quad \bar{w}_{1}^{k}=\prod_{i=1}^{n} \bar{\mu}_{1} \widetilde{F}_{i}^{k}(x), \\
\underline{\phi}_{1}^{k}(x)=\frac{\underline{w}_{1}^{k}(x)}{D_{l}^{1}}, \\
\bar{\phi}_{1}^{k}(x)=\frac{\bar{w}_{1}^{k}}{D_{r}^{1}}, \\
z_{1}^{k}(x)=\sum_{i=1}^{n}{ }^{1} c_{i}^{k} x_{i}+{ }^{1} c_{0}^{k}, \quad k=1, \ldots, M, \\
f_{2}(x)=\gamma \widehat{y}_{l}^{2}(x)+(1-\gamma) \widehat{y}_{r}^{2}(x),
\end{gathered}
$$

where

$$
\begin{aligned}
& \widehat{y}_{l}^{2}(x)=\sum_{k=1}^{M} \underline{\phi}_{2}^{k}(x) z_{2}^{k}(x)=\frac{\sum_{k=1}^{M_{2}} \underline{w}_{2}^{k}(x) z_{2}^{k}(x)}{D_{l}^{2}}, \\
& \widehat{y}_{r}^{2}(x)=\sum_{k=1}^{M_{2}} \bar{\phi}_{2}^{k}(x) z_{2}^{k}(x)=\frac{\sum_{k=1}^{M_{1}} \bar{w}_{2}^{k}(x) z_{2}^{k}(x)}{D_{r}^{2}},
\end{aligned}
$$

where

$$
\begin{gathered}
\underline{w}_{2}^{k}=\prod_{i=1}^{n} \underline{\mu}_{2} \widetilde{F}_{i}^{k}(x), \quad \bar{w}_{2}^{k}=\prod_{i=1}^{n} \bar{\mu}_{2} \widetilde{F}_{i}^{k}(x), \\
D_{l}^{2}=\sum_{k=1}^{M_{2}} \prod_{i=1}^{n} \underline{\mu}_{2} \widetilde{F}_{i}^{k}(x), \quad D_{r}^{2}=\sum_{k=1}^{M_{2}} \prod_{i=1}^{n} \bar{\mu}_{2} \widetilde{F}_{i}^{k}(x), \\
\underline{\phi}_{2}^{k}(x)=\frac{\underline{w}_{2}^{k}(x)}{D_{l}^{2}}, \quad \bar{\phi}_{2}^{k}(x)=\frac{\bar{w}_{2}^{k}(x)}{D_{r}^{2}}, \\
z_{2}^{k}(x)=\sum_{i=1}^{n} c_{i} c_{i}^{k} x_{i}+{ }^{2} c_{0}^{k}, \quad k=1, \ldots, M .
\end{gathered}
$$

Lemma 3. $Y$ is closed under addition.

Proof. The proof of this lemma requires our IT2FNN-0 to be able to approximate sums of functions. Suppose we have 


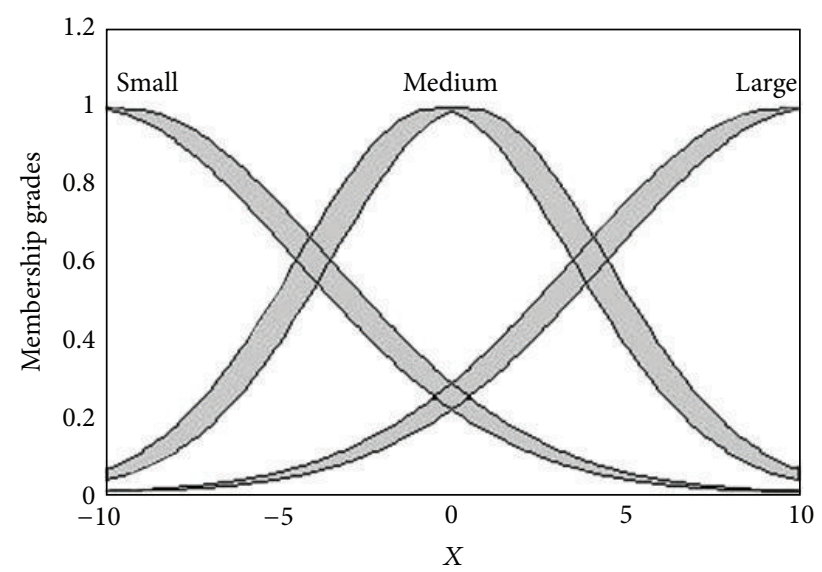

(a) Antecedent IT2MFs for fuzzy rules

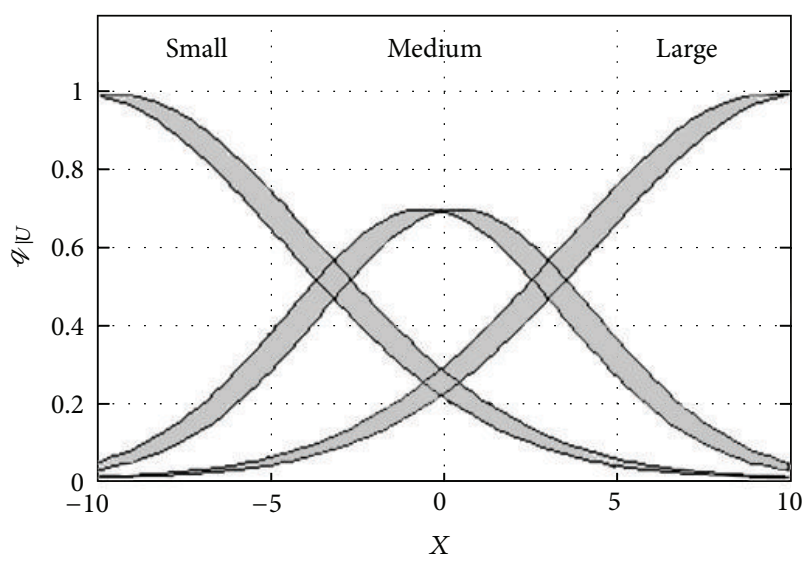

(c) An example of the interval type-2 fuzzy basis functions

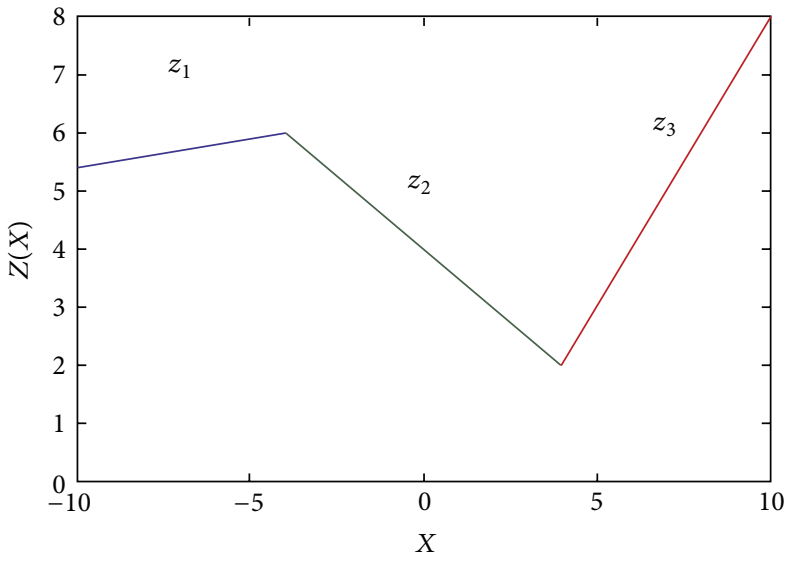

(b) Overall I/O curve for crisp rules

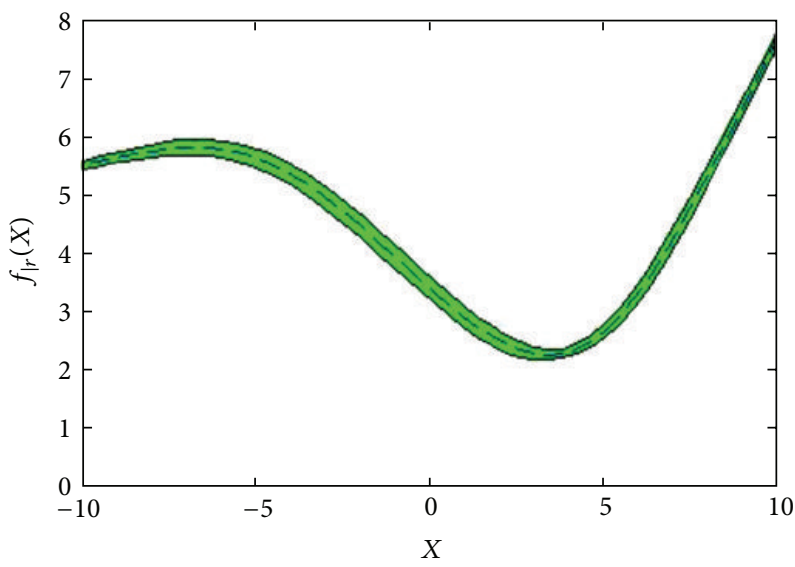

(d) Overall I/O curve for IT2FNN-0

Figure 5: An example of the IT2FNN-0 architecture.

two IT2FNN-0s, $f_{1}(x)$ and $f_{2}(x)$ with $M_{1}$ and $M_{2}$ rules, respectively. The output of each system can be expressed as

$$
\begin{aligned}
f_{1}(x) & +f_{2}(x) \\
= & \frac{\sum_{k_{1}=1}^{M_{1}} \sum_{k_{2}=1}^{M_{2}}\left[\underline{w}_{1}^{k_{1}} \underline{w}_{2}^{k_{2}}\left(\alpha z_{1}^{k_{1}}+\gamma z_{2}^{k_{2}}\right)\right]}{D_{l}^{1} D_{l}^{2}} \\
& +\frac{\sum_{k_{1}=1}^{M_{1}} \sum_{k_{2}=1}^{M_{2}}\left[\bar{w}_{1}^{k_{1}} \bar{w}_{2}^{k_{2}}\left\{(1-\alpha) z_{1}^{k_{1}}+(1-\gamma) z_{2}^{k_{2}}\right\}\right]}{D_{r}^{1} D_{r}^{2}}
\end{aligned}
$$

and that $\underline{\Phi}_{k_{1}, k_{2}}=\left(\underline{w}_{1}^{k_{1}} \underline{w}_{2}^{k_{2}}\right) /\left(D_{r}^{1} D_{r}^{2}\right)$ and $\bar{\Phi}_{k_{1}, k_{2}}=\left(\bar{w}_{1}^{k_{1}} \bar{w}_{2}^{k_{2}}\right) /$ $\left(D_{r}^{1} D_{r}^{2}\right)$, where the FBFs are known to be nonlinear. Therefore, an equivalent to IT2FNN-0 can be constructed under the addition of $f_{1}(x)$ and $f_{2}(x)$, where the consequents form an addition of $\alpha z_{1}^{k_{1}}+\gamma z_{2}^{k_{2}}$ and $(1-\alpha) z_{1}^{k_{1}}+(1-\gamma) z_{2}^{k_{2}}$ multiplied by a respective FBFs expansion (Theorem 1 ), and there exists $f \in Y$ such that $\sup _{x \in U}(|g(x)-f(x)|)<\varepsilon$ (Theorem 2). Since $f(x)$ satisfies Lemma 3 and $Y \in f(x)=f_{1}(x)+f_{2}(x)$ then we can conclude that $Y$ is closed under addition. Note that $z_{1}^{k_{1}}$ and $z_{2}^{k_{2}}$ can be linear since the FBFs are a nonlinear basis interval and therefore the resultant function, $f(x)$, is nonlinear interval (see Figure 5).

Lemma 4. $Y$ is closed under multiplication.

Proof. Similar to Lemma 3, we model the product of $f_{1}(x) f_{2}(x)$ of two IT2FNN-0s. The product $f_{1}(x) f_{2}(x)$ can be expressed as

$$
\begin{aligned}
f_{1}(x) & f_{2}(x) \\
= & \frac{1}{D_{l}^{1} D_{l}^{2}}\left[\sum_{k_{1}=1}^{M_{1}} \sum_{k_{2}=1}^{M_{2}} \underline{w}_{1}^{k_{1}} \underline{w}_{2}^{k_{2}} \alpha \gamma z_{1}^{k_{1}} z_{2}^{k_{2}}\right] \\
& +\frac{1}{D_{l}^{1} D_{r}^{2}}\left[\sum_{k_{1}=1}^{M_{1}} \sum_{k_{2}=1}^{M_{2}} \underline{w}_{1}^{k_{1}} \bar{w}_{2}^{k_{2}} \alpha(1-\gamma) z_{1}^{k_{1}} z_{2}^{k_{2}}\right] \\
& +\frac{1}{D_{r}^{1} D_{l}^{2}}\left[\sum_{k_{1}=1}^{M_{1}} \sum_{k_{2}=1}^{M_{2}} \underline{w}_{1}^{k_{1}} \underline{w}_{2}^{k_{2}}(1-\alpha) \gamma z_{1}^{k_{1}} z_{2}^{k_{2}}\right] \\
& +\frac{1}{D_{r}^{1} D_{r}^{2}}\left[\sum_{k_{1}=1}^{M_{1}} \sum_{k_{2}=1}^{M_{2}} \bar{w}_{1}^{k_{1}} \bar{w}_{2}^{k_{2}}(1-\alpha)(1-\gamma) z_{1}^{k_{1}} z_{2}^{k_{2}}\right] .
\end{aligned}
$$


Therefore, an equivalent to IT2FNN-0 can be constructed under the multiplication of $f_{1}(x)$ and $f_{2}(x)$, where the consequents form an addition of $\alpha \gamma z_{1}^{k_{1}} z_{2}^{k_{2}}, \alpha(1-\gamma) z_{1}^{k_{1}} z_{2}^{k_{2}},(1-$ a) $\gamma z_{1}^{k_{1}} z_{2}^{k_{2}}$, and $(1-\alpha)(1-\gamma) z_{1}^{k_{1}} z_{2}^{k_{2}}$ multiplied by a respective $\operatorname{FBFs}\left(\Phi_{k 1, k 2}^{l, l}=\left(\underline{w}_{1}^{k_{1}} \underline{w}_{2}^{k_{2}}\right) /\left(D_{l}^{1} D_{l}^{2}\right), \Phi_{k 1, k 2}^{l, r}=\left(\underline{w}_{1}^{k_{1}} \bar{w}_{2}^{k_{2}}\right) /\left(D_{l}^{1} D_{r}^{2}\right)\right.$, $\Phi_{k 1, k 2}^{r, l}=\left(\bar{w}_{1}^{k_{1}} \underline{w}_{2}^{k_{2}}\right) /\left(D_{r}^{1} D_{l}^{2}\right)$, and $\left.\Phi_{k 1, k 2}^{r, r}=\left(\bar{w}_{1}^{k_{1}} \bar{w}_{2}^{k_{2}}\right) /\left(D_{r}^{1} D_{r}^{2}\right)\right)$ expansion (Theorem 1), and there exists $f \in Y$ such that $\sup _{x \in U}(|g(x)-f(x)|)<\varepsilon$ (Theorem 2). Since $f(x)$ satisfies Lemma 3 and $Y \in f(x)=f_{1}(x) f_{2}(x)$ then we can conclude that $Y$ is closed under multiplication. Note that $z_{1}^{k_{1}}$ and $z_{2}^{k_{2}}$ can be linear since the FBFs are a nonlinear basis interval and therefore the resultant function, $f(x)$, is nonlinear interval. Also, even if $z_{1}^{k_{1}}$ and $z_{2}^{k_{2}}$ were linear, their product $z_{1}^{k_{1}} z_{2}^{k_{2}}$ is evidently polynomial (see Figure 5).

\section{Lemma 5. $Y$ is closed under scalar multiplication.}

Proof. Let an arbitrary IT2FNN-0 be $f(x)(20)$; the scalar multiplication of $c f(x)$ can be expressed as

$$
\begin{aligned}
c f(x) & =\alpha c \hat{y}_{l}(x)+(1-\alpha) c \hat{y}_{r}(x) \\
& =\frac{\sum_{k=1}^{M}\left[\alpha D_{r} \underline{w}^{k}(x)+(1-\alpha) D_{l} \bar{w}^{k}(x)\right] c z^{k}(x)}{D_{l} D_{r}} .
\end{aligned}
$$

Therefore we can construct an IT2FNN-0 that computes $c z^{k}(x)$ in the form of the proposed IT2FNN-0; $Y$ is closed under scalar multiplication.

Lemma 6. For every $\mathbf{x}^{0}, \mathbf{y}^{0} \in U$ and $\mathbf{x}^{0} \neq \mathbf{y}^{0}$, there exists $f \in Y$ such that $f\left(\mathbf{x}^{0}\right) \neq f\left(\mathbf{y}^{0}\right)$; that is, $Y$ separates points on $U$.

Proof. We prove that $\left(Y, d_{\infty}\right)$ separates points on $U$. We prove this by constructing a required $f(x)(20)$; that is, we specify $f \in Y$ such that $f\left(\mathbf{x}^{0}\right) \neq f\left(\mathbf{y}^{0}\right)$ for arbitrarily given $\left(\mathbf{x}^{0}, \mathbf{y}^{0}\right) \epsilon$ $U$ with $\mathbf{x}^{0} \neq \mathbf{y}^{0}$. We choose two fuzzy rules in the form of (8) for the fuzzy rule base (i.e., $M=2)$. Let $x^{0}=\left(x_{1}^{0}, x_{2}^{0}, \ldots, x_{n}^{0}\right)$ and $y^{0}=\left(y_{1}^{0}, y_{2}^{0}, \ldots, y_{n}^{0}\right)$. If $x_{i}^{0}=\left(x_{l i}^{0}+x_{r i}^{0}\right) / 2$ and $y_{i}^{0}=\left(y_{l i}^{0}+\right.$ $\left.y_{r i}^{0}\right) / 2$ with $x_{i}^{0} \neq y_{i}^{0}$, we define two interval type-2 fuzzy sets $\left(\widetilde{F}_{i}^{1},\left[\underline{\mu}_{\widetilde{F}_{i}^{i}}, \bar{\mu}_{\widetilde{F}_{i}^{1}}\right]\right)$ and $\left(\widetilde{F}_{i}^{2},\left[\underline{\mu}_{\widetilde{F}_{i}^{2}}, \bar{\mu}_{\widetilde{F}_{i}^{2}}\right]\right)$ with

$$
\begin{gathered}
\bar{\mu}_{\widetilde{F}_{i}^{1}}\left(x_{i}\right)= \begin{cases}\exp \left[-\frac{1}{2}\left(x_{i}-x_{l i}^{0}\right)^{2}\right], & x_{i}<x_{l i}^{0}, \\
1, & x_{l i}^{0} \leq x_{i} \leq x_{r i}^{0}, \\
\exp \left[-\frac{1}{2}\left(x_{i}-x_{r i}^{0}\right)^{2}\right], & x_{i}>x_{l i}^{0},\end{cases} \\
\underline{\mu}_{\widetilde{F}_{i}^{1}}\left(x_{i}\right)= \begin{cases}\exp \left[-\frac{1}{2}\left(x_{i}-x_{r i}^{0}\right)^{2}\right], & x_{i} \leq x_{l i}^{0}, \\
\exp \left[-\frac{1}{2}\left(x_{i}-x_{l i}^{0}\right)^{2}\right], & x_{i}>x_{r i}^{0},\end{cases}
\end{gathered}
$$

$$
\begin{gathered}
\bar{\mu}_{\widetilde{F}_{i}}\left(x_{i}\right)= \begin{cases}\exp \left[-\frac{1}{2}\left(x_{i}-y_{l i}^{0}\right)^{2}\right], & x_{i}<y_{l i}^{0}, \\
1, & y_{l i}^{0} \leq x_{i} \leq y_{r i}^{0}, \\
\exp \left[-\frac{1}{2}\left(x_{i}-y_{r i}^{0}\right)^{2}\right], & x_{i}>y_{l i}^{0},\end{cases} \\
\underline{\mu}_{\widetilde{F}_{i}^{k}}\left(x_{i}\right)= \begin{cases}\exp \left[-\frac{1}{2}\left(x_{i}-y_{r i}^{0}\right)^{2}\right], & x_{i} \leq y_{l i}^{0}, \\
\exp \left[-\frac{1}{2}\left(x_{i}-y_{l i}^{0}\right)^{2}\right], & x_{i}>y_{r i}^{0} .\end{cases}
\end{gathered}
$$

If $x_{i}^{0}=y_{i}^{0}$, then $\widetilde{F}_{i}^{1}=\widetilde{F}_{i}^{2}$ and $\underline{\mu}_{\widetilde{F}_{i}^{1}}\left(x_{i}^{0}\right)=\underline{\mu}_{\widetilde{F}_{i}^{2}}\left(y_{i}^{0}\right), \bar{\mu}_{\widetilde{F}_{i}^{1}}\left(x_{i}^{0}\right)=$ $\bar{\mu}_{\widetilde{F}^{2}}\left(y_{i}^{0}\right)$; that is, only one interval type-2 fuzzy set is defined. We define two real value sets $z^{1}$ and $z^{2}$ with $z^{k}(x)=$ $\sum_{i=1}^{n} c_{i}^{k} x_{i}+c_{0}^{k}$, where $k=1,2$. Now we have specified all the design parameters except $z^{k}$; that is, we have already obtained a function $f$ which is in the form of (10) with $M=2$ and $\left(\widetilde{F}_{i}^{1},\left[\underline{\mu}_{\widetilde{F}_{i}^{1}}, \bar{\mu}_{\widetilde{F}_{i}^{1}}\right]\right)$ given by (18), (20), and (21). With this $f$, we have

$$
\begin{aligned}
f\left(x^{0}\right)= & \alpha\left[\underline{\phi}^{1}\left(x^{0}\right) z^{1}\left(x^{0}\right)+\underline{\phi}^{2}\left(x^{0}\right) z^{2}\left(x^{0}\right)\right]+(1-\alpha) \\
& \times\left[\bar{\phi}^{1}\left(x^{0}\right) z^{1}\left(x^{0}\right)+\left(1-\bar{\phi}^{1}\left(x^{0}\right)\right) z^{2}\left(x^{0}\right)\right],
\end{aligned}
$$

where

$$
\begin{gathered}
\underline{\phi}^{1}\left(x^{0}\right)=\frac{\prod_{i=1}^{n} \underline{\mu}_{F_{i}^{1}}\left(x_{i}^{0}\right)}{\prod_{i=1}^{n} \underline{\mu}_{F_{i}^{1}}\left(x_{i}^{0}\right)+\prod_{i=1}^{n} \underline{\mu}_{F_{i}^{2}}\left(x_{i}^{0}\right)}, \\
\underline{\phi}^{2}\left(x^{0}\right)=\frac{\prod_{i=1}^{n} \underline{\mu}_{F_{i}^{2}}\left(x_{i}^{0}\right)}{\prod_{i=1}^{n} \underline{\mu}_{F_{i}^{1}}\left(x_{i}^{0}\right)+\prod_{i=1}^{n} \underline{\mu}_{F_{i}^{2}}\left(x_{i}^{0}\right)}, \\
\bar{\phi}^{1}\left(x^{0}\right)=\frac{1}{1+\prod_{i=1}^{n} \bar{\mu}_{F_{i}^{2}}\left(x_{i}^{0}\right)}, \\
f\left(y^{0}\right)=\alpha\left[\underline{\phi}^{1}\left(y^{0}\right) z^{1}\left(y^{0}\right)+\underline{\phi}^{2}\left(y^{0}\right) z^{2}\left(y^{0}\right)\right]+(1-\alpha) \\
\times\left[\left(1-\bar{\phi}^{2}\left(y^{0}\right)\right) z^{1}\left(y^{0}\right)+\bar{\phi}^{2}\left(y^{0}\right) z^{2}\left(y^{0}\right)\right],
\end{gathered}
$$

where

$$
\begin{gathered}
\underline{\phi}^{1}\left(y^{0}\right)=\frac{\prod_{i=1}^{n} \underline{\mu}_{F_{i}^{1}}\left(y_{i}^{0}\right)}{\prod_{i=1}^{n} \underline{\mu}_{F_{i}^{1}}\left(y_{i}^{0}\right)+\prod_{i=1}^{n} \underline{\mu}_{F_{i}^{2}}\left(y_{i}^{0}\right)}, \\
\underline{\phi}^{2}\left(y^{0}\right)=\frac{\prod_{i=1}^{n} \underline{\mu}_{F_{i}^{2}}\left(y_{i}^{0}\right)}{\prod_{i=1}^{n} \underline{\mu}_{F_{i}^{1}}\left(y_{i}^{0}\right)+\prod_{i=1}^{n} \underline{\mu}_{F_{i}^{2}}\left(y_{i}^{0}\right)}, \\
\bar{\phi}^{2}\left(y^{0}\right)=\frac{1}{\prod_{i=1}^{n} \bar{\mu}_{F_{i}^{1}}\left(y_{i}^{0}\right)+1} .
\end{gathered}
$$

Since $\mathbf{x}^{0} \neq \mathbf{y}^{0}$, there must be some $i$ such that $x_{i}^{0}=y_{i}^{0}$; hence, we have $\prod_{i=1}^{n} \underline{\mu}_{F_{i}^{1}}\left(x_{i}^{0}\right) \neq 1$ and $\prod_{i=1}^{n} \bar{\mu}_{F_{i}^{2}}\left(x_{i}^{0}\right) \neq 1$. If we choose 
$z^{1}=0$ and $z^{2}=1$, then $f\left(x^{0}\right)=\alpha \phi^{2}\left(x^{0}\right)+(1-\alpha)\left[1-\bar{\phi}^{1}\left(x^{0}\right)\right] \neq$ $\alpha \phi^{2}\left(y^{0}\right)+(1-\alpha) \bar{\phi}^{2}\left(y^{0}\right)=f\left(y^{0}\right)$. Separability is satisfied whenever an IT2FNN-0 can compute strictly monotonic functions of each input variable. This can easily be achieved by adjusting the membership functions of the premise part. Therefore, $\left(Y, d_{\infty}\right)$ separates points on $U$.

Lemma 7. For each $x \in U$, there exists $f \in Y$ such that $f(x) \neq$ 0 ; that is, $Y$ vanishes at no point of $U$.

Finally, we prove that $\left(Y, d_{\infty}\right)$ vanishes at no point of $U$. By observing (8)-(11), (20), and (21), we simply choose all $y^{k}(x)>0(k=1,2, \ldots, M)$; that is, any $f \in Y$ with $y^{k}(x)>0$ serves as the required $f$.

Proof of Theorem 2. From (20) and (21), it is evident that $Y$ is a set of real continuous functions on $U$, which are established by using complete interval type-2 fuzzy sets in the IF parts of fuzzy rules. Using Lemmas 3, 4, and 5, Y is proved to be an algebra. By using the Stone-Weierstrass theorem together with Lemmas 6 and 7, we establish that the proposed IT2FNN-0 possesses the universal approximation capability.

3.3. Applying the Stone-Weierstrass Theorem to the IT2FNN-2 Architecture. We now consider a subset of the IT2FNN-2 on Figure 2. The set of IT2FNN-2 with singleton fuzzifier, product inference, type-reduction defuzzifier (KM) [13, 14], and Gaussian interval type-2 membership function consists of all FBF expansion functions. $f: U \subset R^{n} \rightarrow R, x=\left(x_{1}, x_{2}, \ldots\right.$, $\left.x_{n}\right) \in U ; \mu_{\widetilde{F}_{i}^{k}(x)} \in\left[\underline{\mu}_{\widetilde{F}_{i}^{k}}(x), \bar{\mu}_{\widetilde{F}_{i}^{k}}(x)\right]$ is the Gaussian interval type-2 membership function, igaussmtype $2\left(x,\left[\sigma_{i}^{k},{ }_{1} m_{i}^{k}\right.\right.$, $\left.\left.{ }^{1} m_{i}^{k}\right]\right)$, defined by (8)-(11). If we view $\phi_{l}^{k}(x), \bar{\phi}_{l}^{k}(x), \underline{\phi}_{r}^{k}(x)$, $\bar{\phi}_{r}^{k}(x)$ as basis functions (44), (46), (49), (50) and $y_{l}^{k}, y_{r}^{k}$ are linear functions (41), then $\hat{y}(x)$ can be viewed as a linear combination of the basis functions. Let $Y$ be the set of all the FBF expansions with $\underline{\phi}_{l}^{k}(x), \bar{\phi}_{l}^{k}(x), \underline{\phi}_{r}^{k}(x), \bar{\phi}_{r}^{k}(x)$ and let $d_{\infty}\left(f_{1}, f_{2}\right)=\sup _{x \in U}\left(\left|f_{1}(x)-f_{2}(x)\right|\right)$ be the supmetric; then, $\left(Y, d_{\infty}\right)$ is a metric space [38]. The following theorem shows that $\left(Y, d_{\infty}\right)$ is dense in $\left(C[U], d_{\infty}\right)$, where $C[U]$ is the set of all real continuous functions defined on $U$. We use the following Stone-Weierstrass theorem to prove the theorem.

Suppose we have two IT2FNN-2s $f_{1}, f_{2} \in Y$; the output of each system can be expressed as

$$
f_{1}(x)=\gamma \widehat{y}_{l}^{1}(x)+(1-\gamma) \hat{y}_{r}^{1}(x),
$$

where

$$
\begin{aligned}
\hat{y}_{l}^{1}(x) & =\sum_{k_{1}=1}^{L_{1}}{ }^{1} \bar{\phi}_{l}^{k_{1}}(x)^{1} z_{l}^{k_{1}}(x)+\sum_{k_{1}=L_{1}+1}^{M_{1}}{ }^{1} \underline{\phi}_{l}^{k_{1}}(x)^{1} z_{l}^{k_{1}}(x) \\
& =\frac{\sum_{k_{1}=1}^{L_{1}} \bar{w}_{1}^{k_{1}}(x)^{1} z_{l}^{k_{1}}(x)+\sum_{k=L_{1}+1}^{M_{1}} \underline{w}_{l}^{k_{1}}(x)^{1} z_{l}^{k_{1}}(x)}{D_{l}^{1}},
\end{aligned}
$$

$$
\begin{aligned}
\hat{y}_{r}^{1}(x) & =\sum_{k_{1}=1}^{R_{1}} \underline{\phi}_{r}^{k_{1}}(x){ }^{1} z_{r}^{k_{1}}(x)+\sum_{k_{1}=R_{1}+1}^{M_{1}}{ }^{1} \bar{\phi}_{r}^{k_{1}}(x){ }^{1} z_{r}^{k_{1}}(x) \\
& =\frac{\sum_{k_{1}=1}^{R_{1}} \underline{w}_{1}^{k_{1}}(x){ }^{1} z_{r}^{k_{1}}(x)+\sum_{k_{1}=R_{1}+1}^{M_{1}} \bar{w}_{1}^{k_{1}}(x)^{1} z_{r}^{k_{1}}(x)}{D_{r}^{1}},
\end{aligned}
$$

where

$$
\begin{aligned}
& \underline{w}_{1}^{k_{1}}=\prod_{i=1}^{n} \underline{\mu}_{1} \tilde{F}_{i}^{k_{1}}(x), \quad \bar{w}_{1}^{k_{1}}=\prod_{i=1}^{n} \bar{\mu}_{1} \tilde{F}_{i}^{k_{1}}(x), \\
& D_{l}^{1}=\sum_{k_{1}=1}^{L_{1}} \bar{w}_{1}^{k_{1}}+\sum_{k_{1}=L_{1}+1}^{M_{1}} \underline{w}_{1}^{k_{1}}, \\
& D_{r}^{1}=\sum_{k_{1}=1}^{R_{1}} \underline{w}_{1}^{k_{1}}+\sum_{k_{1}=R_{1}+1}^{M_{1}} \bar{w}_{1}^{k_{1}}, \\
& { }^{1} \bar{\phi}_{l}^{k_{1}}(x)=\frac{\bar{w}_{1}^{k_{1}}}{D_{l}^{1}} \quad \forall k_{1}=1, \ldots, L_{1}(x), \\
& { }^{1} \underline{\phi}_{l}^{k_{1}}(x)=\frac{w_{1}^{k_{1}}}{D_{l}^{1}} \quad \forall k_{1}=L_{1}(x)+1, \ldots, M_{1} \text {, } \\
& { }^{1} \underline{\phi}_{r}^{k_{1}}(x)=\frac{\underline{w}_{1}^{k_{1}}}{D_{r}^{1}} \quad \forall k_{1}=1, \ldots, R_{1}(x), \\
& { }^{1} \bar{\phi}_{r}^{k_{1}}(x)=\frac{\bar{w}_{1}^{k_{1}}}{D_{r}^{1}} \quad \forall k_{1}=R_{1}(x)+1, \ldots, M_{1}, \\
& { }^{1} z_{l}^{k_{1}}=\sum_{i=1}^{n}{ }^{1} c_{i}^{k_{1}} x_{i}+{ }^{1} c_{0}^{k}-\sum_{i=1}^{n}{ }^{1} s_{i}^{k_{1}}\left|x_{i}\right|-{ }^{1} s_{0}^{k_{1}}, \\
& k_{1}=1, \ldots, M_{1} \text {, } \\
& { }^{1} z_{r}^{k_{1}}=\sum_{i=1}^{n}{ }^{1} c_{i}^{k_{1}} x_{i}+{ }^{1} c_{0}^{k_{1}}+\sum_{i=1}^{n}{ }^{1} s_{i}^{k_{1}}\left|x_{i}\right|+{ }^{1} s_{0}^{k_{1}} \text {, } \\
& k_{1}=1, \ldots, M_{1}, \\
& f_{2}(x)=\gamma \widehat{y}_{l}^{2}(x)+(1-\gamma) \hat{y}_{r}^{2}(x) \text {, }
\end{aligned}
$$

where

$$
\begin{aligned}
& \hat{y}_{l}^{2}(x) \\
& =\sum_{k_{2}=1}^{L_{2}} \bar{\phi}_{l}^{k_{2}}(x)^{2} z_{l}^{k_{2}}(x)+\sum_{k_{2}=L_{2}+1}^{M_{2}}{ }^{2} \underline{\phi}_{l}^{k_{2}}(x)^{2} z_{l}^{k_{2}}(x) \\
& =\frac{\sum_{k_{2}=1}^{L_{2}} \bar{w}_{2}^{k_{2}}(x)^{2} z_{l}^{k_{2}}(x)+\sum_{k_{2}=L_{2}+1}^{M_{2}} \underline{w}_{2}^{k_{2}}(x)^{2} z_{l}^{k_{2}}(x)}{D_{l}^{2}},
\end{aligned}
$$




$$
\begin{aligned}
& \hat{y}_{r}^{2}(x) \\
& =\sum_{k_{2}=1}^{R_{2}} \underline{\phi}_{r}^{k_{2}}(x)^{2} z_{r}^{k_{2}}(x)+\sum_{k_{2}=R_{2}+1}^{M_{2}}{ }^{2} \bar{\phi}_{r}^{k_{2}}(x)^{2} z_{r}^{k_{2}}(x) \\
& =\frac{\sum_{k_{2}=1}^{R_{21}} \underline{w}_{2}^{k_{2}}(x)^{2} z_{r}^{k_{2}}(x)+\sum_{k_{2}=R_{2}+1}^{M_{2}} \bar{w}_{2}^{k_{2}}(x)^{2} z_{r}^{k_{2}}(x)}{D_{r}^{1}},
\end{aligned}
$$

where

$$
\begin{gathered}
\underline{w}_{2}^{k_{2}}=\prod_{i=1}^{n} \underline{\mu}_{2} \tilde{F}_{i}^{k_{2}}(x), \quad \bar{w}_{2}^{k_{2}}=\prod_{i=1}^{n} \underline{\mu}_{2} \tilde{F}_{i}^{k_{2}}(x), \\
D_{l}^{2}=\sum_{k_{2}=1}^{L_{2}} \bar{w}_{2}^{k_{2}}+\sum_{k_{2}=L_{2}+1}^{M_{2}} \underline{w}_{2}^{k_{2}}, \\
D_{r}^{2}=\sum_{k_{2}=1}^{R_{2}} \underline{w}_{2}^{k_{2}}+\sum_{k_{2}=R_{2}+1}^{M_{2}} \bar{w}_{2}^{k_{2}}, \\
{ }^{2} \bar{\phi}_{l}^{k_{2}}(x)=\frac{\bar{w}_{2}^{k_{2}}}{D_{l}^{2}} \quad \forall k_{2}=1, \ldots, L_{2}(x), \\
{ }^{2} \underline{\phi}_{l}^{k_{2}}(x)=\frac{\underline{w}_{2}^{k_{2}}}{D_{l}^{2}} \quad \forall k_{2}=L_{2}(x)+1, \ldots, M_{2}, \\
\underline{\phi}_{r}^{k_{2}}(x)=\frac{\underline{w}_{2}^{k_{2}}}{D_{r}^{2}} \quad \forall k_{2}=1, \ldots, R_{2}(x), \\
{ }^{2} \phi_{r}^{k_{2}}(x)=\frac{\bar{w}_{2}^{k_{2}}}{D_{r}^{2}} \quad \forall k_{2}=R_{2}(x)+1, \ldots, M_{2}, \\
{ }^{2} z_{l}^{k_{2}}=\sum_{i=1}^{n} c_{i}^{k_{2}} x_{i}+{ }^{2} c_{0}^{k_{2}}-\sum_{i=1}^{n}{ }^{2} s_{i}^{k_{2}}\left|x_{i}\right|-{ }^{2} s_{0}^{k_{2}}, \\
z_{r}^{k_{2}}=\sum_{i=1}^{n} c_{i}^{2} c_{i_{2}}^{k_{2}} x_{i}+{ }^{2} c_{0}^{k_{2}}+\sum_{i=1}^{n} s_{i}^{k_{2}}\left|x_{i}\right|+{ }^{2} s_{0}^{k_{2}}, \\
k_{2}=1, \ldots, M_{2},
\end{gathered}
$$

\section{Lemma 8. $Y$ is closed under addition.}

Proof. The proof of this lemma requires our IT2FNN-2 to be able to approximate sums of functions. Suppose we have two IT2FNN-2s $f_{1}(x)$ and $f_{2}(x)$ with rules $M_{1}$ and $M_{2}$, respectively. The output of each system can be expressed as

$$
\begin{aligned}
& f_{1}(x)+f_{2}(x) \\
& =\left(\left(\sum_{k_{1}=1}^{L_{1}} \sum_{k_{2}=1}^{L_{2}}\left[\alpha D_{l}^{2} \bar{w}_{1}^{k_{1} 1} z_{l}^{k_{1}}(x)+\gamma D_{l}^{1} \bar{w}_{2}^{k_{2}} z_{l}^{k_{2}}(x)\right]\right)\right. \\
& \left.\quad \times\left(D_{l}^{1} D_{l}^{2}\right)^{-1}\right)
\end{aligned}
$$

$$
\begin{aligned}
& +\left(\left(\sum_{k_{1}=L_{1}+1}^{M_{1}} \sum_{k_{2}=L_{2}+1}^{M_{2}}\left[\alpha D_{l}^{2} \underline{w}_{1}^{k_{1} 1} z_{l}^{k_{1}}(x)+\gamma D_{l}^{1} \underline{w}_{2}^{k_{2} 2} z_{l}^{k_{2}}(x)\right]\right)\right. \\
& \left.\times\left(D_{l}^{1} D_{l}^{2}\right)^{-1}\right) \\
& +\left(\left(\sum _ { k _ { 1 } = 1 } ^ { R _ { 1 } } \sum _ { k _ { 2 } = 1 } ^ { R _ { 2 } } \left[(1-\alpha) D_{r}^{2} \underline{w}_{1}^{k_{1} 1} z_{r}^{k_{1}}(x)\right.\right.\right. \\
& +\left(\left(\sum_{k_{1}=R_{1}+1}^{M_{1}} \sum_{k_{2}=R_{2}+1}^{M_{2}}\left[(1-\alpha) D_{r}^{1} \underline{w}_{2}^{k_{2} 2} z_{r}^{k_{2}}(x)\right]\right) \times\left(D_{r}^{1} D_{r}^{2}\right)^{-1} z_{r}^{k_{1}}(x)\right. \\
& \left.\left.+(1-\gamma) D_{r}^{1} \bar{w}_{2}^{k_{2} 2} z_{r}^{k_{2}}(x)\right]\right) \\
& \left.\quad \times\left(D_{r}^{1} D_{r}^{2}\right)^{-1}\right) .
\end{aligned}
$$

Therefore, an equivalent to IT2FNN-2 can be constructed under the addition of $f_{1}(x)$ and $f_{2}(x)$, where the consequents form an addition of $\alpha^{1} z_{l}^{k_{1}}+\gamma^{2} z_{l}^{k_{2}}$ and $(1-\alpha)^{1} z_{r}^{k_{1}}+(1-\gamma)^{2} z_{r}^{k_{2}}$ multiplied by a respective FBFs expansion (Theorem 1), and there exists $f \in Y$ such that $\sup _{x \in U}(|g(x)-f(x)|)<\varepsilon$ (Theorem 2). Since $f(x)$ satisfies Lemma 3 and $Y \in f(x)=$ $f_{1}(x)+f_{2}(x)$ then we can conclude that $Y$ is closed under addition. Note that $z_{1}^{k_{1}}$ and $z_{2}^{k_{2}}$ can be linear interval since the FBFs are a nonlinear basis and therefore the resultant function, $f(x)$, is nonlinear interval (see Figure 6).

Lemma 9. $Y$ is closed under multiplication.

Proof. In a similar way to Lemma 8 , we model the product of $f_{1}(x) f_{2}(x)$ of two IT2FNN-2s which is the last point we need to demonstrate before we can conclude that the StoneWeierstrass theorem can be applied to the proposed reasoning mechanism. The product $f_{1}(x) f_{2}(x)$ can be expressed as

$$
\begin{aligned}
& f_{1}(x) f_{2}(x) \\
& =\frac{\alpha \gamma}{D_{l}^{1} D_{l}^{2}} \\
& \quad \times\left[\sum_{k_{1}=1}^{L_{1}} \sum_{k_{2}=1}^{L_{2}} \frac{w_{1}^{k_{1}}}{(x)} \bar{w}_{2}^{k_{2}}(x){ }^{1} z_{l}^{k_{1}}(x)^{2} z_{l}^{k_{2}}(x)\right. \\
& \quad+\sum_{k_{1}=1}^{L_{1}} \sum_{k_{2}=L_{2}+1}^{M_{2}} \bar{w}_{1}^{k_{1}}(x) \underline{w}_{2}^{k_{2}}(x){ }^{1} z_{l}^{k_{1}}(x)^{2} z_{l}^{k_{2}}(x) \\
& \quad+\sum_{k_{1}=L_{1}+1}^{M_{1}} \sum_{k_{2}=1}^{L_{2}} \underline{w}_{1}^{k_{1}}(x) \bar{w}_{2}^{k_{2}}(x){ }^{1} z_{l}^{k_{1}}(x)^{2} z_{l}^{k_{2}}(x)
\end{aligned}
$$




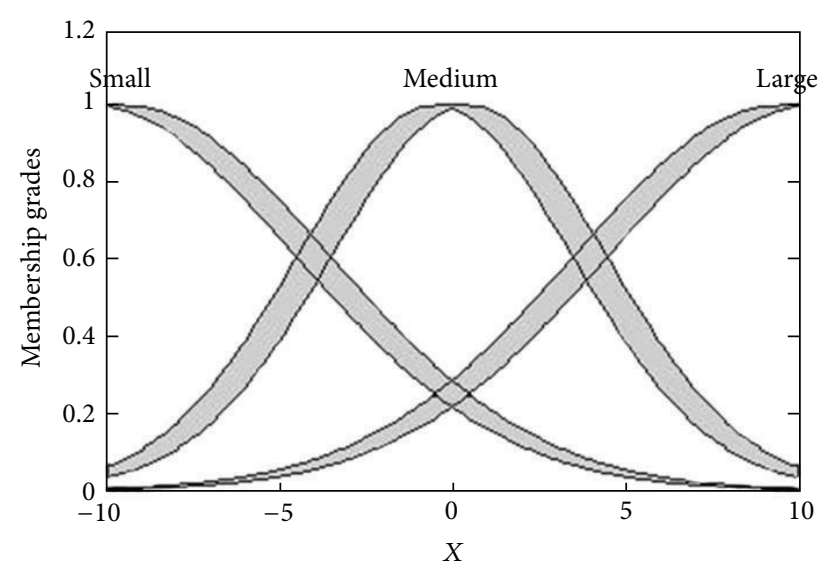

(a) Antecedent IT2MFs for fuzzy rules

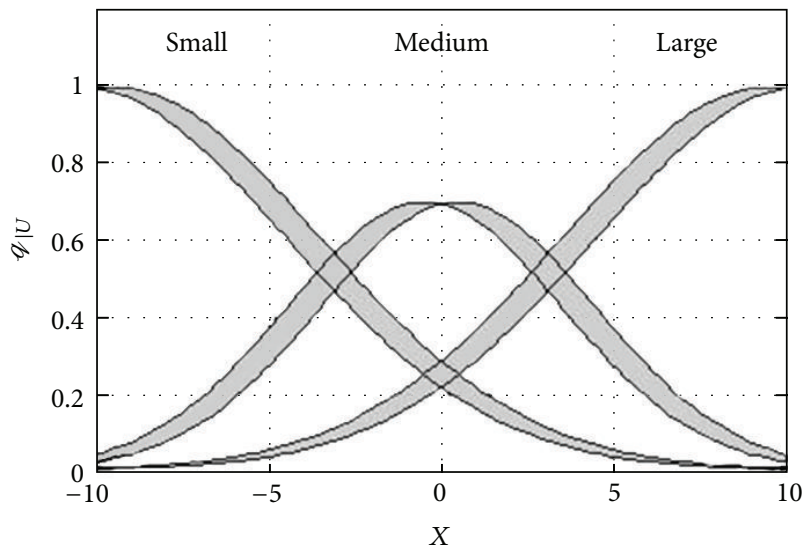

(c) An example of the interval type-2 fuzzy basis functions

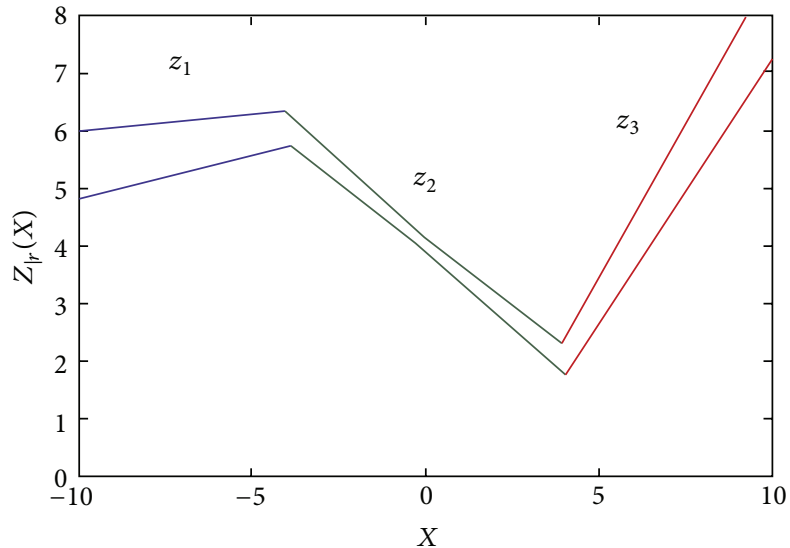

(b) Overall I/O curve for interval rules

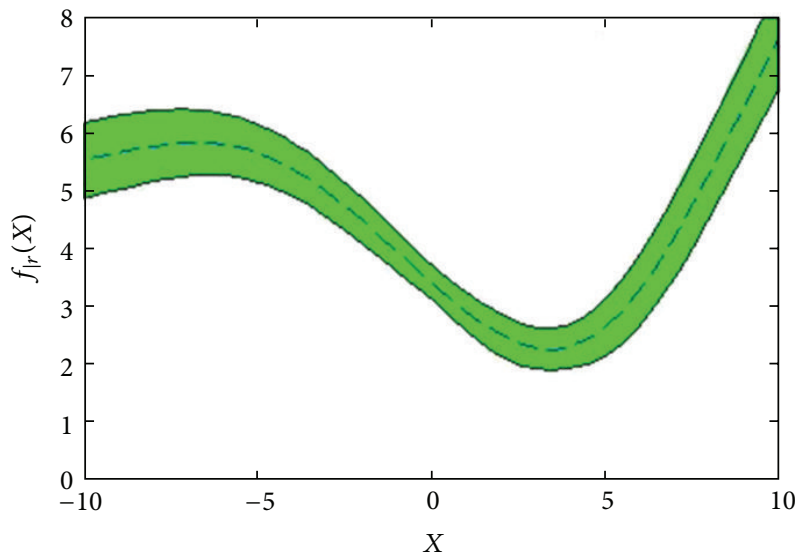

(d) Overall I/O curve for IT2FNN-2

FIGURE 6: An example of the IT2FNN-2 architecture.

$$
\begin{aligned}
& \left.+\sum_{k_{1}=L_{1}+1}^{M_{1}} \sum_{k_{2}=L_{2}+1}^{M_{2}} \underline{w}_{1}^{k_{1}}(x) \underline{w}_{2}^{k_{2}}(x){ }^{1} z_{l}^{k_{1}}(x){ }^{2} z_{l}^{k_{2}}(x)\right] \\
& \times\left[\sum_{k_{1}=1}^{R_{1}} \sum_{k_{2}=1}^{L_{2}} \underline{w}_{1}^{k_{1}}(x) \bar{w}_{2}^{k_{2}}(x){ }^{1} z_{r}^{k_{1}}(x){ }^{2} z_{l}^{k_{2}}(x)\right. \\
& +\frac{\alpha(1-\gamma)}{D_{l}^{1} D_{r}^{2}} \\
& +\sum_{k_{1}=1}^{R_{1}} \sum_{k_{2}=L_{2}+1}^{M_{2}} \underline{w}_{1}^{k_{1}}(x) \underline{w}_{2}^{k_{2}}(x){ }^{1} z_{r}^{k_{1}}(x)^{2} z_{l}^{k_{2}}(x) \\
& \times\left[\sum_{k_{1}=1}^{L_{1}} \sum_{k_{2}=1}^{R_{2}} \bar{w}_{1}^{k_{1}}(x) \underline{w}_{2}^{k_{2}}(x){ }^{1} z_{l}^{k_{1}}(x)^{2} z_{r}^{k_{2}}(x)\right. \\
& +\sum_{k_{1}=R_{1}+1}^{M_{1}} \sum_{k_{2}=1}^{L_{2}} \bar{w}_{1}^{k_{1}}(x) \bar{w}_{2}^{k_{2}}(x){ }^{1} z_{r}^{k_{1}}(x)^{2} z_{l}^{k_{2}}(x) \\
& +\sum_{k_{1}=1}^{L_{1}} \sum_{k_{2}=R_{2}+1}^{M_{2}} \bar{w}_{1}^{k_{1}}(x) \bar{w}_{2}^{k_{2}}(x){ }^{1} z_{l}^{k_{1}}(x)^{2} z_{r}^{k_{2}}(x) \\
& +\sum_{k_{1}=L_{1}+1}^{M_{1}} \sum_{k_{2}=1}^{R_{2}} \underline{w}_{1}^{k_{1}}(x) \underline{w}_{2}^{k_{2}}(x){ }^{1} z_{l}^{k_{1}}(x)^{2} z_{r}^{k_{2}}(x) \\
& \left.+\sum_{k_{1}=L_{1}+1}^{M_{1}} \sum_{k_{2}=R_{2}+1}^{M_{2}} \underline{w}_{1}^{k_{1}}(x) \bar{w}_{2}^{k_{2}}(x){ }^{1} z_{l}^{k_{1}}(x)^{2} z_{r}^{k_{2}}(x)\right] \\
& \left.+\sum_{k_{1}=R_{1}+1}^{M_{1}} \sum_{k_{2}=R_{2}+1}^{M_{2}} \bar{w}_{1}^{k_{1}}(x) \underline{w}_{2}^{k_{2}}(x){ }^{1} z_{r}^{k_{1}}(x)^{2} z_{l}^{k_{2}}(x)\right] \\
& +\frac{(1-\alpha)(1-\gamma)}{D_{r}^{1} D_{r}^{2}} \\
& \times\left[\sum_{k_{1}=1}^{R_{1}} \sum_{k_{2}=1}^{R_{2}} \underline{w}_{1}^{k_{1}}(x) \underline{w}_{2}^{k_{2}}(x){ }^{1} z_{r}^{k_{1}}(x)^{2} z_{r}^{k_{2}}(x)\right. \\
& +\frac{(1-\alpha) \gamma}{D_{r}^{1} D_{l}^{2}} \\
& +\sum_{k_{1}=1}^{R_{1}} \sum_{k_{2}=R_{2}+1}^{M_{2}} \underline{w}_{1}^{k_{1}}(x) \bar{w}_{2}^{k_{2}}(x){ }^{1} z_{r}^{k_{1}}(x)^{2} z_{r}^{k_{2}}(x)
\end{aligned}
$$




$$
\begin{aligned}
& +\sum_{k_{1}=R_{1}+1}^{M_{1}} \sum_{k_{2}=1}^{R_{2}} \bar{w}_{1}^{k_{1}}(x) \underline{w}_{2}^{k_{2}}(x){ }^{1} z_{r}^{k_{1}}(x)^{2} z_{r}^{k_{2}}(x) \\
& \left.+\sum_{k_{1}=R_{1}+1}^{M_{1}} \sum_{k_{2}=R_{2}+1}^{M_{2}} \bar{w}_{1}^{k_{1}}(x) \bar{w}_{2}^{k_{2}}(x){ }^{1} z_{r}^{k_{1}}(x)^{2} z_{r}^{k_{2}}(x)\right] .
\end{aligned}
$$

Therefore, an equivalent to IT2FNN-2 can be constructed under the multiplication of $f_{1}(x)$ and $f_{2}(x)$, where the consequents form an addition of $\alpha{ }^{1} z_{l}^{k_{1}} z_{l}^{k_{2}}, \alpha(1-\gamma)^{1} z_{l}^{k_{1}{ }^{2}} z_{r}^{k_{2}}$, $(1-\alpha) \gamma^{1} z_{r}^{k_{1}} z_{l}^{k_{2}}$, and $(1-\alpha)(1-\gamma){ }^{1} z_{r}^{k_{1}} z_{r}^{k_{2}}$ multiplied by a respective FBFs expansion (Theorem 1 ), and there exists $f \in Y$ such that $\sup _{x \in U}(|g(x)-f(x)|)<\varepsilon$ (Theorem 2). Since $f(x)$ satisfies Lemma 3 and $Y \in f(x)=f_{1}(x) f_{2}(x)$ then we can conclude that $Y$ is closed under multiplication. Note that $z_{1}^{k_{1}}$ and $z_{2}^{k_{2}}$ can be linear intervals since the FBFs are a nonlinear basis interval and therefore the resultant function, $f(x)$, is nonlinear interval. Also, even if $z_{1}^{k_{1}}$ and $z_{2}^{k_{2}}$ were linear, their product $z_{1}^{k_{1}} z_{2}^{k_{2}}$ is evidently polynomial interval (see Figure 10).

\section{Lemma 10. $Y$ is closed under scalar multiplication.}

Proof. Let an arbitrary IT2FNN-2 be $f(x)$ (51); the scalar multiplication of $c f(x)$ can be expressed as

$$
\begin{aligned}
c f_{1}(x) & \\
= & \alpha c \widehat{y}_{l}^{1}(x)+(1-\alpha) c \widehat{y}_{r}^{1}(x) \\
= & \frac{\sum_{k_{1}=1}^{L_{1}} \bar{w}_{1}^{k_{1}}(x) \alpha c^{1} z_{l}^{k_{1}}(x)+\sum_{k=L_{1}+1}^{M_{1}} \underline{w}_{1}^{k_{1}}(x) \alpha c^{1} z_{l}^{k_{1}}(x)}{D_{l}^{1}} \\
& +\frac{\sum_{k_{1}=1}^{R_{1}} \bar{w}_{1}^{k_{1}}(x)(1-\alpha) c^{1} z_{r}^{k_{1}}(x)}{D_{r}^{1}} \\
& +\frac{\sum_{k_{1}=R_{1}+1}^{M_{1}} \underline{w}_{1}^{k_{1}}(x)(1-\alpha) c^{1} z_{r}^{k_{1}}(x)}{D_{r}^{1}} .
\end{aligned}
$$

Therefore we can construct an IT2FNN-2 that computes all FBF expansion combinations with $\alpha c^{1} z_{l}^{k_{1}}(x)$ and (1 $\alpha) c^{1} z_{r}^{k_{1}}(x)$ in the form of the proposed IT2FNN-2, and $Y$ is closed under scalar multiplication.

Lemma 11. For every $\left(\mathbf{x}^{0}, \mathbf{y}^{0}\right) \in U$ and $\mathbf{x}^{0} \neq \mathbf{y}^{0}$, there exists $f \in$ $Y$ such that $f\left(\mathbf{x}^{0}\right) \neq f\left(\mathbf{y}^{0}\right)$; that is, $Y$ separates points on $U$.

We prove this by constructing a required $f$; that is, we specify $f \in Y$ such that $f\left(\mathbf{x}^{0}\right) \neq f\left(\mathbf{y}^{0}\right)$ for arbitrarily given $\mathbf{x}^{0}, \mathbf{y}^{0} \in U$ with $\mathbf{x}^{0} \neq \mathbf{y}^{0}$. We choose two fuzzy rules in the form of (8) for the fuzzy rule base (i.e., $M=2$ ). Let $x^{0}=$ $\left(x_{1}^{0}, x_{2}^{0}, \ldots, x_{n}^{0}\right)$ and $y^{0}=\left(y_{1}^{0}, y_{2}^{0}, \ldots, y_{n}^{0}\right)$. If $x_{i}^{0}=\left(x_{l i}^{0}+x_{r i}^{0}\right) / 2$ and $y_{i}^{0}=\left(y_{l i}^{0}+y_{r i}^{0}\right) / 2$ with $x_{i}^{0} \neq y_{i}^{0}$, we define two interval type-2 fuzzy sets $\left(\widetilde{F}_{i}^{1},\left[\underline{\mu}_{\widetilde{F}_{i}^{1}}, \bar{\mu}_{\widetilde{F}_{i}^{1}}\right]\right)$ and $\left(\widetilde{F}_{i}^{2},\left[\underline{\mu}_{\widetilde{F}_{i}^{2}}, \bar{\mu}_{\widetilde{F}_{i}^{2}}\right]\right)$ with

$$
\begin{gathered}
\bar{\mu}_{\widetilde{F}_{i}^{1}}\left(x_{i}\right)= \begin{cases}\exp \left[-\frac{1}{2}\left(x_{i}-x_{l i}^{0}\right)^{2}\right], & x_{i}<x_{l i}^{0}, \\
1, & x_{l i}^{0} \leq x_{i} \leq x_{r i}^{0}, \\
\exp \left[-\frac{1}{2}\left(x_{i}-x_{r i}^{0}\right)^{2}\right], & x_{i}>x_{l i}^{0},\end{cases} \\
\underline{\mu}_{\widetilde{F}_{i}^{1}}\left(x_{i}\right)= \begin{cases}\exp \left[-\frac{1}{2}\left(x_{i}-x_{r i}^{0}\right)^{2}\right], & x_{i} \leq x_{l i}^{0}, \\
\exp \left[-\frac{1}{2}\left(x_{i}-x_{l i}^{0}\right)^{2}\right], & x_{i}>x_{r i}^{0},\end{cases} \\
\bar{\mu}_{\widetilde{F}_{i}^{2}}\left(x_{i}\right)= \begin{cases}\exp \left[-\frac{1}{2}\left(x_{i}-y_{l i}^{0}\right)^{2}\right], & x_{i}<y_{l i}^{0}, \\
1, & y_{l i}^{0} \leq x_{i} \leq y_{r i}^{0}, \\
\exp \left[-\frac{1}{2}\left(x_{i}-y_{r i}^{0}\right)^{2}\right], & x_{i}>y_{l i}^{0},\end{cases} \\
\underline{\mu}_{\tilde{F}_{i}^{k}}\left(x_{i}\right)= \begin{cases}\exp \left[-\frac{1}{2}\left(x_{i}-y_{r i}^{0}\right)^{2}\right], & x_{i} \leq y_{l i}^{0}, \\
\exp \left[-\frac{1}{2}\left(x_{i}-y_{l i}^{0}\right)^{2}\right], & x_{i}>y_{r i}^{0} .\end{cases}
\end{gathered}
$$

If $x_{i}^{0}=y_{i}^{0}$, then $\widetilde{F}_{i}^{1}=\widetilde{F}_{i}^{2}$ and $\underline{\mu}_{\widetilde{F}_{i}^{1}}\left(x_{i}^{0}\right)=\underline{\mu}_{\widetilde{F}_{i}^{2}}\left(y_{i}^{0}\right), \bar{\mu}_{\widetilde{F}_{i}^{1}}\left(x_{i}^{0}\right)=$ $\bar{\mu}_{\tilde{F}_{i}^{2}}\left(y_{i}^{0}\right)$; that is, only one interval type-2 fuzzy set is defined. We define two interval value real sets $z^{1} \in\left[z_{l}^{1}, z_{r}^{1}\right]$ and $z^{2} \in$ $\left[z_{l}^{2}, z_{r}^{2}\right]$. Now we have specified all the design parameters except $\left[z_{l}^{k}, z_{r}^{k}\right]$; that is, we have already obtained a function $f$ which is in the form of (20), (21) with $M=2$ and $\left.\left(\widetilde{F}_{i}^{k}, \underline{\mu}_{\widetilde{F}_{i}^{k}}, \bar{\mu}_{\widetilde{F}_{i}^{k}}\right]\right)$ given by (8)-(11). With this $f$, we have

$$
\begin{aligned}
f\left(x^{0}\right)= & \alpha\left[\phi_{l}^{1}\left(x^{0}\right) z_{l}^{1}\left(x^{0}\right)+\left(1-\phi_{l}^{1}\left(x^{0}\right)\right) z_{l}^{2}\left(x^{0}\right)\right] \\
& +(1-\alpha)\left[\phi_{r}^{1}\left(x^{0}\right) z_{r}^{1}\left(x^{0}\right)+\phi_{r}^{2}\left(x^{0}\right) z_{r}^{2}\left(x^{0}\right)\right],
\end{aligned}
$$

where

$$
\begin{gathered}
\phi_{l}^{1}\left(x^{0}\right)=\frac{1}{1+\prod_{i=1}^{n} \underline{\mu}_{F_{i}^{2}}\left(x_{i}^{0}\right)}, \\
\phi_{r}^{1}\left(x^{0}\right)=\frac{\prod_{i=1}^{n} \underline{\mu}_{F_{i}^{1}}\left(x_{i}^{0}\right)}{\prod_{i=1}^{n} \underline{\mu}_{F_{i}^{1}}\left(x_{i}^{0}\right)+\prod_{i=1}^{n} \bar{\mu}_{F_{i}^{2}}\left(x_{i}^{0}\right)}, \\
\phi_{r}^{2}\left(x^{0}\right)=\frac{\prod_{i=1}^{n} \bar{\mu}_{F_{i}^{2}}\left(x_{i}^{0}\right)}{\prod_{i=1}^{n} \underline{\mu}_{F_{i}^{1}}\left(x_{i}^{0}\right)+\prod_{i=1}^{n} \bar{\mu}_{F_{i}^{2}}\left(x_{i}^{0}\right)}, \\
f\left(y^{0}\right)=\alpha\left[\phi_{l}^{1}\left(y^{0}\right) z_{l}^{1}\left(y^{0}\right)+\phi_{l}^{2}\left(y^{0}\right) z_{l}^{2}\left(y^{0}\right)\right]+(1-\alpha) \\
\times\left[\left(1-\phi_{r}^{2}\left(y^{0}\right)\right) z_{r}^{1}\left(y^{0}\right)+\phi_{r}^{2}\left(y^{0}\right) z_{r}^{2}\left(y^{0}\right)\right],
\end{gathered}
$$

where

$$
\phi_{r}^{2}\left(y^{0}\right)=\frac{1}{\prod_{i=1}^{n} \underline{\mu}_{F_{i}^{1}}\left(y_{i}^{0}\right)+1},
$$




$$
\begin{aligned}
\phi_{l}^{1}\left(y^{0}\right) & =\frac{\prod_{i=1}^{n} \bar{\mu}_{F_{i}^{1}}\left(y_{i}^{0}\right)}{\prod_{i=1}^{n} \bar{\mu}_{F_{i}^{1}}\left(y_{i}^{0}\right)+\prod_{i=1}^{n} \underline{\mu}_{F_{i}^{2}}\left(y_{i}^{0}\right)}, \\
\phi_{l}^{2}\left(y^{0}\right) & =\frac{\prod_{i=1}^{n} \underline{\mu}_{F_{i}^{2}}\left(y_{i}^{0}\right)}{\prod_{i=1}^{n} \bar{\mu}_{F_{i}^{1}}\left(y_{i}^{0}\right)+\prod_{i=1}^{n} \underline{\mu}_{F_{i}^{2}}\left(y_{i}^{0}\right)} .
\end{aligned}
$$

Since $\mathbf{x}^{0} \neq \mathbf{y}^{0}$, there must be some $\mathbf{i}$ such that $x_{i}^{0}=y_{i}^{0}$; hence, we have $\prod_{i=1}^{n} \underline{\mu}_{F_{i}^{1}}\left(x_{i}^{0}\right) \neq 1$ and $\prod_{i=1}^{n} \bar{\mu}_{F_{i}^{2}}\left(x_{i}^{0}\right) \neq 1$. If we choose $z_{l r}^{1}=0$ and $z_{l r}^{2}=1$, then $f\left(x^{0}\right)=\alpha\left(1-\phi_{l}^{1}\left(x^{0}\right)\right)+$ $(1-\alpha) \phi_{r}^{2}\left(x^{0}\right) \neq \alpha \phi_{l}^{2}\left(y^{0}\right)+(1-\alpha) \phi_{r}^{2}\left(y^{0}\right)=f\left(y^{0}\right)$. Therefore, $\left(Y, d_{\infty}\right)$ separates point on $U$.

Lemma 12. For each $x \in U$, there exists $f \in Y$ such that $f(x) \neq 0$; that is, $Y$ vanishes at no point of $U$.

Finally, we prove that $\left(Y, d_{\infty}\right)$ vanishes at no point of $U$. By observing (8)-(11), (20), and (21), we just choose all $z^{k}>0$ $(k=1,2, \ldots, M)$; that is, any $f \in Y$ with $z^{k}>0$ serves as required $f$.

Proof of Theorem 2. From (20) and (21), it is evident that $Y$ is a set of real continuous functions on $U$, which are established by using complete interval type- 2 fuzzy sets in the IF parts of fuzzy rules. Using Lemmas 8,9 , and $10, Y$ is proved to be an algebra. By using the Stone-Weierstrass theorem together with Lemmas 11 and 12, we establish that the proposed IT2FNN-2 possesses the universal approximation capability.

Therefore by choosing appropriate class of interval type- 2 membership functions, we can conclude that the IT2FNN-0 and IT2FNN-2 with simplified fuzzy if-then rules satisfy the five criteria of the Stone-Weierstrass theorem.

\section{Application Examples}

In this section the results from simulations using ANFIS, IT2FNN-0, IT2FNN-1 [35], IT2FNN-2, and IT2FNN-3 [35] are presented for nonlinear system identification and forecasting the Mackey-Glass chaotic time series [39] with $\tau=$ 60 with different signal noise ratio values, $\operatorname{SNR}(\mathrm{dB})=0,10$, 20,30 , free as uncertainty source. These examples are used as benchmark problems to test the proposed ideas in the paper. We have to mention that the IT2FNN-1 and IT2FNN3 architectures are very similar to I2FNN-0 and IT2FNN2 , respectively [35], and their results are presented for comparison purposes. The proposed IT2FNN architectures are validated using 10-fold cross-validation [40, 41] considering sum of square errors (SSE) or root mean square error (RMSE) in the training or test phase. We use cross-validation to measure the variability of the RMSE in the training and testing phases to compare network architectures IT2FNN. Cross-validation procedure evaluation is done using Matlab's crossvalind function. Noise is added by Matlab's awgn function.

In $K$-fold cross-validation [40], the original sample is randomly partitioned into $K$ subsamples. Of the $K$ subsamples,
TABLE 1: RMSE (CHK) values of ANFIS and IT2FNN with 10-fold cross-validation for identifying non-linearity of Experiment 1.

\begin{tabular}{lccccc}
\hline SNR (dB) ANFIS & IT2FNN-0 & IT2FNN-1 & IT2FNN-2 & IT2FNN-3 \\
\hline 0 & 0.6156 & 0.3532 & 0.2764 & 0.2197 & 0.1535 \\
10 & 0.2375 & 0.1153 & 0.0986 & 0.0683 & 0.0453 \\
20 & 0.0806 & 0.0435 & 0.0234 & 0.0127 & 0.0087 \\
30 & 0.0225 & 0.0106 & 0.0079 & 0.0045 & 0.0028 \\
Free & 0.0015 & 0.0009 & 0.0004 & 0.0002 & 0.0001 \\
\hline
\end{tabular}

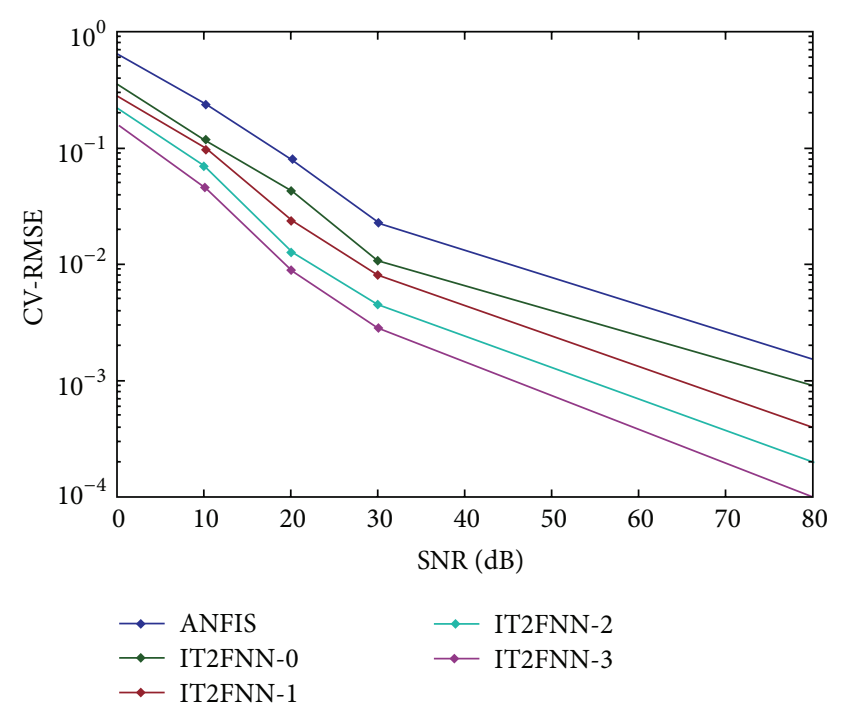

FIgURE 7: RMSE (CHK) values of ANFIS and IT2FNN using 10-fold cross-validation for identifying nonlinearity in Experiment 1.

a single subsample is retained as the validation data for testing the model, and the remaining $K-1$ subsamples are used as training data. The cross-validation process is then repeated $K$ times (the folds), with each of the $K$ subsamples used exactly once as the validation data. The $K$ results from the folds then can be averaged (or otherwise combined) to produce a single estimation. The advantage of this method over repeated random subsampling is that all observations are used for both training and validation, and each observation is used for validation exactly once. 10 -fold cross-validation is commonly used. Three application examples are used to illustrate proofs of universality, as follows.

Experiment 1 (identification of a one variable nonlinear function). In this experiment we approximate a nonlinear function $f: \mathfrak{R} \rightarrow \mathfrak{R}$ :

$$
f(u)=0.6 \sin (\pi u)+0.3 \sin (3 \pi u)+0.1 \sin (5 \pi u)+\eta,
$$

(where $\eta$ is a uniform noise component) using a-one input one-output IT2FNN, 50 training data sets with 10-fold cross-validation with uniform noise levels, six IT2MFs type igaussmtype2, 6 rules, and 50 epochs. Once the ANFIS and IT2FNN models are identified a comparison was made, taking into account RMSE statistic values with 10-fold crossvalidation. Table 1 and Figure 7 show the resulting RMSE 
TABLE 2: Resulting RMSE (CHK) values in ANFIS and IT2FNN for non-linearity identification in Experiment 2 with 10-fold crossvalidation.

\begin{tabular}{|c|c|c|c|c|c|}
\hline SNR (dB & ANFIS & IT2FNN-0 & IT2FNN-1 & IT2FNN-2 & IT2FNN-3 \\
\hline 0 & 1.0432 & 0.7203 & 0.6523 & 0.5512 & 0.5267 \\
\hline 10 & 0.3096 & 0.2798 & 0.2583 & 0.2464 & 0.2344 \\
\hline 20 & 0.1703 & 0.1637 & 0.1592 & 0.1465 & 0.1387 \\
\hline 30 & 0.1526 & 0.1408 & 0.1368 & 0.1323 & 0.1312 \\
\hline Free & 0.1503 & 0.1390 & 0.1323 & 0.1304 & 0.1276 \\
\hline
\end{tabular}

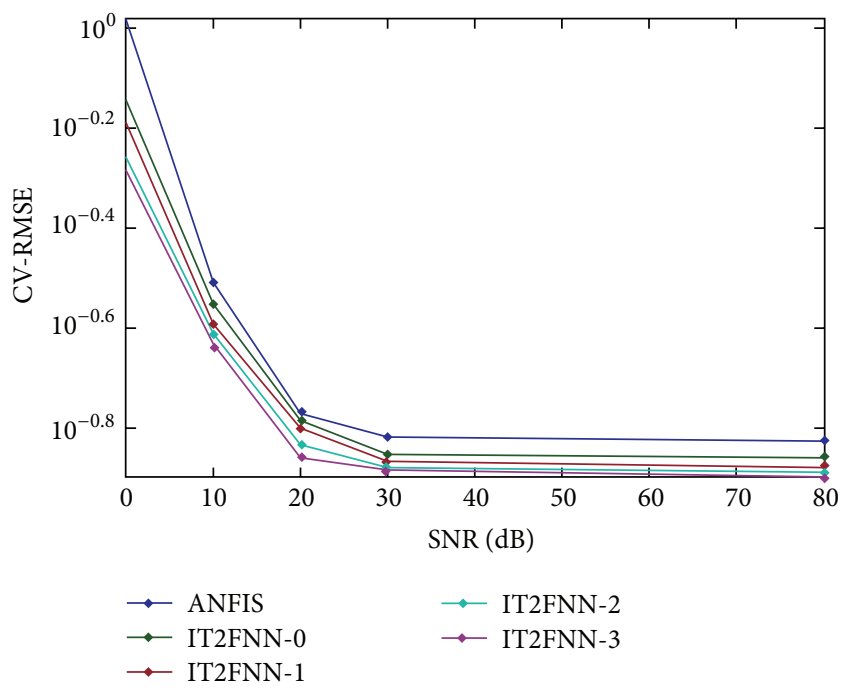

FIGURE 8: Resulting RMSE (CHK) values obtained by ANFIS and IT2FNN for nonlinearity identification in Experiment 2 with 10 -fold cross-validation.

(CHK) values for ANFIS and IT2FNN; it can be seen that IT2FNN architectures [31] perform better than ANFIS.

Experiment 2 (identification of a three variable nonlinear function). A three-input one-output IT2FNN is used to approximate nonlinear Sugeno [27] function $f: \mathfrak{R}^{3} \rightarrow \mathfrak{R}$ :

$$
f\left(x_{1}, x_{2}, x_{3}\right)=\left(1+\sqrt{x_{1}}+\frac{1}{x_{2}}+\frac{1}{\sqrt{x_{3}^{3}}}\right)^{2}+\eta
$$

216 training data sets are generated with 10 -fold crossvalidation and 125 for tests; 2 igaussmtype2 IT2MFs for each input, 8 rules, and 50 epochs. Once the ANFIS and IT2FNN models are identified, a comparison is made with RMSE statistic values and 10 -fold cross-validation. Table 2 and Figure 8 show the resultant RMSE (CHK) values for ANFIS and IT2FNN. It can be seen that IT2FNN architectures [31] perform better than ANFIS.

Experiment 3. Predicting the Mackey-Glass chaotic time series.

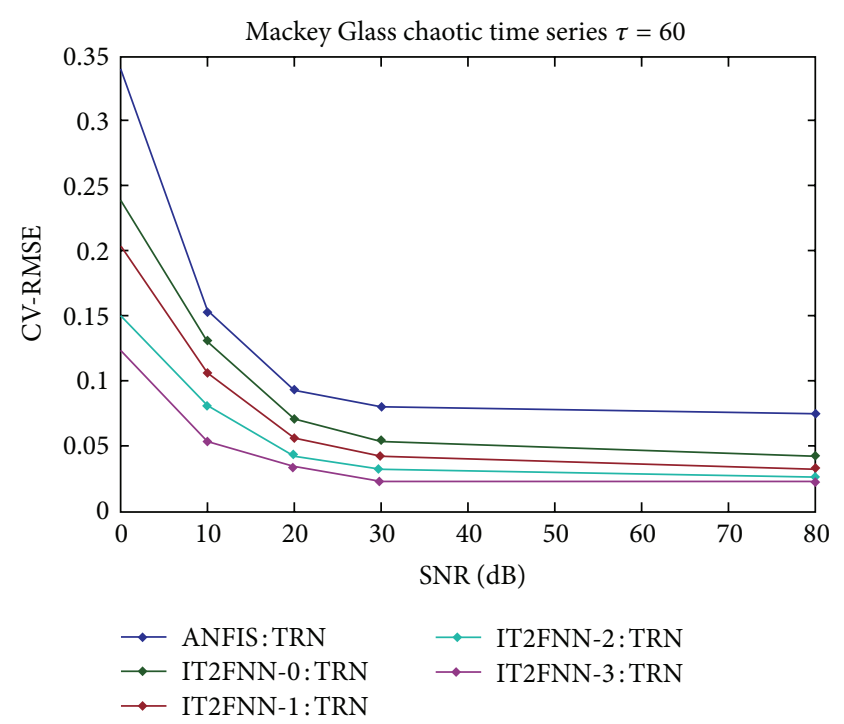

FIGURE 9: RMSE (TRN) values determined by ANFIS and IT2FNN models with 10 -fold cross-validation for Mackey-Glass chaotic time series prediction with $\tau=60$.

Mackey-Glass chaotic time series is a well-known benchmark [39] for systems modeling and is described as follows:

$$
\dot{x}(t)=\frac{0.1 x(t-\tau)}{1+x^{10}(t-\tau)}-0.1 x(t) .
$$

1200 data sets are generated based on initial conditions $x(0)=$ 1.2 and $\tau=60$, using fourth order Runge-Kutta method adding different levels of uniform noise. For comparing with other methods, an input-output vector is chosen for IT2FNN model with the following format:

$$
[x(t-18), x(t-12), x(t-6), x(t) ; x(t+6)] .
$$

Four-input and one-output IT2FNN model is used for Mackey-Glass chaotic time series prediction, choosing 500 data sets for training and 500 test data data sets with 10fold cross-validation test, 2 IT2MFs for each input with membership function igaussmtype 2,16 rules, and 50 epochs. ANFIS and IT2FNN models are identified, comparing RMSE statistical values with 10 -fold cross-validation. Table 3 and Figures 9 and 10 show the number of $\varsigma$ points out of uncertainty interval $\tilde{Y}(x) \in\left[\widehat{y}_{l}(x), \widehat{y}_{r}(x)\right]$ evaluated by IT2FNN model, RMSE training values (TRN) and test (CHK) obtained for ANFIS and IT2FNN models. It can be seen that IT2FNN model architectures predict better Mackey-Glass chaotic time series.

\section{Conclusions}

In this paper we have shown that an interval type-2 fuzzy neural network (IT2FNN) is a universal approximator. Simulation results of nonlinear function identification using 
TABLE 3: RMSE (TRN/CHK) and $\varsigma$ values determined by ANFIS and IT2FNN models with 10 fold cross-validation for Mackey-Glass chaotic time series prediction with $\tau=60$.

\begin{tabular}{|c|c|c|c|c|c|c|c|c|c|c|c|c|c|c|c|}
\hline \multirow{2}{*}{$\begin{array}{l}\text { SNR }(\mathrm{dB}) \\
\tau=60\end{array}$} & \multicolumn{3}{|c|}{ ANFIS } & \multicolumn{3}{|c|}{ IT2FNN-0 } & \multicolumn{3}{|c|}{ IT2FNN-1 } & \multicolumn{3}{|c|}{ IT2FNN-2 } & \multicolumn{3}{|c|}{ IT2FNN-3 } \\
\hline & TRN & $\mathrm{CHK}$ & $\varsigma$ & TRN & $\mathrm{CHK}$ & $\varsigma$ & TRN & $\mathrm{CHK}$ & $\varsigma$ & TRN & $\mathrm{CHK}$ & $\varsigma$ & TRN & $\mathrm{CHK}$ & $\varsigma$ \\
\hline 0 & 0.3403 & 0.3714 & NA & 0.2388 & 0.2687 & 37 & 2027 & 0.2135 & 35 & .1495 & 0.1536 & 34 & 0.1244 & 0.1444 & 31 \\
\hline 10 & 0.1544 & 0.1714 & NA & 0.1312 & .1456 & 33 & 1069 & 0.1119 & 30 & .0805 & 0.0972 & 28 & 0.0532 & 0.0629 & 25 \\
\hline 20 & 0.0929 & 0.1007 & NA & 0.0708 & 0.0781 & 28 & 0.0566 & 0.0594 & 26 & 0.0424 & 0.0485 & 24 & 0.0342 & 0.0355 & 21 \\
\hline 30 & 788 & 0.0847 & NA & 0.0526 & 0.0582 & 19 & 0.0411 & 0.0468 & 18 & 0.0309 & 0.0332 & 16 & 0.0227 & 0.0316 & 14 \\
\hline Free & 0.0749 & 0.0799 & NA & 0.0414 & 0.0477 & 10 & 0.0321 & 0.0353 & 9 & 0.0251 & 0.0319 & 6 & 0.0215 & 0.0293 & 4 \\
\hline
\end{tabular}

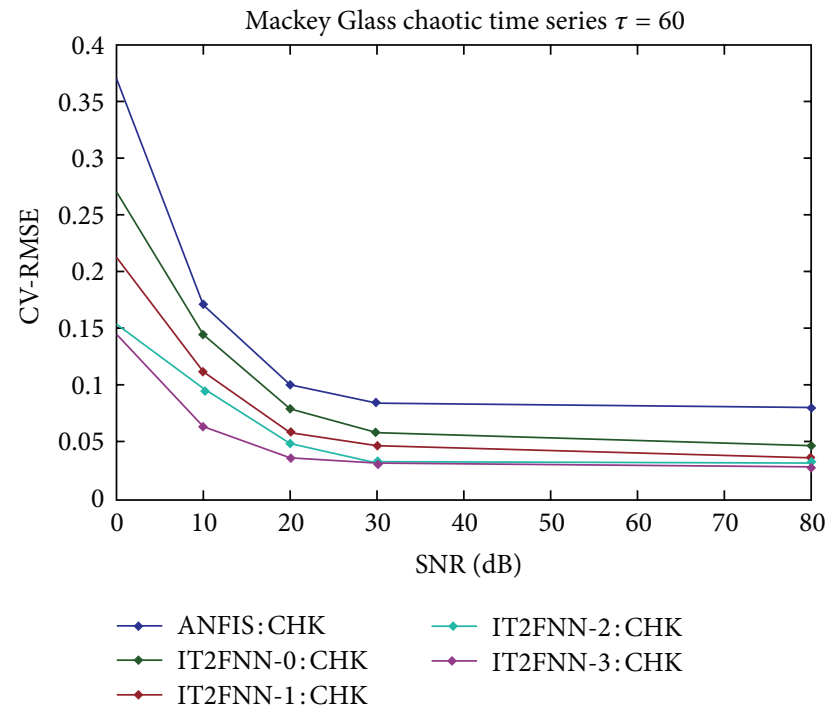

FIGURE 10: RMSE (CHK) values determined by ANFIS and IT2FNN models with 10 -fold cross-validation for Mackey-Glass chaotic time series prediction with $\tau=60$.

the IT2FNN for one and three variables and for the MackeyGlass chaotic time series prediction have been presented to illustrate the theoretical result. In these experiments, the estimated RMSE values for nonlinear function identification with 10-fold cross-validation for the hybrid architectures (IT2FNN-2:A2C0 and IT2FNN-2:A2C1) illustrate the proof based on Stone-Weierstrass theorem, that they are universal approximators for efficient identification of nonlinear functions, complying with $|g(x)-f(x)|<\varepsilon$. Also, it can be seen that while increasing the Signal Noise Ratio (SNR), IT2FNN architectures handle uncertainty more efficiently. We have also illustrated the ideas presented in the paper with the benchmark problem of Mackey-Glass chaotic time series prediction.

\section{Acknowledgments}

The authors would like to thank CONACYT and DGEST for the financial support given for this research project.
The student J. R. Castro was supported by a scholarship from MYCDI, UABC-CONACYT.

\section{References}

[1] L.-X. Wang and J. M. Mendel, "Fuzzy basis functions, universal approximation, and orthogonal least-squares learning," IEEE Transactions on Neural Networks, vol. 3, no. 5, pp. 807-814, 1992.

[2] B. Kosko, "Fuzzy systems as universal approximators," IEEE Transactions on Computers, vol. 43, no. 11, pp. 1329-1333, 1994.

[3] J. J. Buckley, “Universal fuzzy controllers," Automatica, vol. 28, no. 6, pp. 1245-1248, 1992.

[4] J. J. Buckley and Y. Hayashi, "Can fuzzy neural nets approximate continuous fuzzy functions?” Fuzzy Sets and Systems, vol. 61, no. 1, pp. 43-51, 1994.

[5] L. V. Kantorovich and G. P. Akilov, Functional Analysis, Pergamon, Oxford, UK, 2nd edition, 1982.

[6] H. L. Royden, Real Analysis, Macmillan, New York, NY, USA, 2nd edition, 1968.

[7] V. Kreinovich, G. C. Mouzouris, and H. T. Nguyen, "Fuzzy rule based modelling as a universal aproximation tool," in Fuzzy Systems, Modeling and Control, H. T. Nguyen and M. Sugeno, Eds., pp. 135-195, Kluwer Academic Publisher, Boston, Mass, USA, 1998.

[8] M. G. Joo and J. S. Lee, "Universal approximation by hierarchical fuzzy system with constraints on the fuzzy rule," Fuzzy Sets and Systems, vol. 130, no. 2, pp. 175-188, 2002.

[9] B. Kosko, Neural Networks and Fuzzy Systems, Prentice Hall, Englewood Cliffs, NJ, USA, 1992.

[10] J.-S. R. Jang, "ANFIS: adaptive-network-based fuzzy inference system," IEEE Transactions on Systems, Man and Cybernetics, vol. 23, no. 3, pp. 665-685, 1993.

[11] S. M. Zhou and L. D. Xu, "A new type of recurrent fuzzy neural network for modeling dynamic systems," KnowledgeBased Systems, vol. 14, no. 5-6, pp. 243-251, 2001.

[12] S. M. Zhou and L. D. Xu, "Dynamic recurrent neural networks for a hybrid intelligent decision support system for the metallurgical industry," Expert Systems, vol. 16, no. 4, pp. 240-247, 1999.

[13] Q. Liang and J. M. Mendel, "Interval type-2 fuzzy logic systems: theory and design," IEEE Transactions on Fuzzy Systems, vol. 8, no. 5, pp. 535-550, 2000.

[14] J. M. Mendel, Uncertain Rule-Based Fuzzy Logic Systems: Introduction and New Directions, Prentice Hall, Upper Sadler River, NJ, USA, 2001.

[15] C.-H. Lee, T.-W. Hu, C.-T. Lee, and Y.-C. Lee, "A recurrent interval type-2 fuzzy neural network with asymmetric membership 
functions for nonlinear system identification," in Proceedings of the IEEE International Conference on Fuzzy Systems (FUZZ '08), pp. 1496-1502, Hong Kong, June 2008.

[16] C. H. Lee, J. L. Hong, Y. C. Lin, and W. Y. Lai, "Type-2 fuzzy neural network systems and learning," International Journal of Computational Cognition, vol. 1, no. 4, pp. 79-90, 2003.

[17] C.-H. Wang, C.-S. Cheng, and T.-T. Lee, "Dynamical optimal training for interval type-2 fuzzy neural network (T2FNN)," IEEE Transactions on Systems, Man, and Cybernetics, Part B, vol. 34, no. 3, pp. 1462-1477, 2004.

[18] J. T. Rickard, J. Aisbett, and G. Gibbon, "Fuzzy subsethood for fuzzy sets of type-2 and generalized type-n," IEEE Transactions on Fuzzy Systems, vol. 17, no. 1, pp. 50-60, 2009.

[19] S.-M. Zhou, J. M. Garibaldi, R. I. John, and F. Chiclana, "On constructing parsimonious type-2 fuzzy logic systems via influential rule selection," IEEE Transactions on Fuzzy Systems, vol. 17, no. 3, pp. 654-667, 2009.

[20] N. S. Bajestani and A. Zare, "Application of optimized type 2 fuzzy time series to forecast Taiwan stock index," in Proceedings of the 2nd International Conference on Computer, Control and Communication, pp. 1-6, February 2009.

[21] B. Karl, Y. Koçyiðit, and M. Korürek, "Differentiating types of muscle movements using a wavelet based fuzzy clustering neural network," Expert Systems, vol. 26, no. 1, pp. 49-59, 2009.

[22] S. M. Zhou, H. X. Li, and L. D. Xu, "A variational approach to intensity approximation for remote sensing images using dynamic neural networks," Expert Systems, vol. 20, no. 4, pp. 163-170, 2003.

[23] S. Panahian Fard and Z. Zainuddin, "Interval type-2 fuzzy neural networks version of the Stone-Weierstrass theorem," Neurocomputing, vol. 74, no. 14-15, pp. 2336-2343, 2011.

[24] K. Hornik, M. Stinchcombe, and H. White, "Multilayer feedforward networks are universal approximators," Neural Networks, vol. 2, no. 5, pp. 359-366, 1989.

[25] L.-X. Wang and J. M. Mendel, "Generating fuzzy rules by learning from examples," IEEE Transactions on Systems, Man and Cybernetics, vol. 22, no. 6, pp. 1414-1427, 1992.

[26] J. J. Buckley, "Sugeno type controllers are universal controllers," Fuzzy Sets and Systems, vol. 53, no. 3, pp. 299-303, 1993.

[27] T. Takagi and M. Sugeno, "Fuzzy identification of systems and its applications to modeling and control," IEEE Transactions on Systems, Man and Cybernetics, vol. 15, no. 1, pp. 116-132, 1985.

[28] L. A. Zadeh, "Fuzzy sets," Information and Control, vol. 8, no. 3, pp. 338-353, 1965.

[29] K. Hirota and W. Pedrycz, "OR/AND neuron in modeling fuzzy set connectives," IEEE Transactions on Fuzzy Systems, vol. 2, no. 2, pp. 151-161, 1994.

[30] J.-S. R. Jang, C. T. Sun, and E. Mizutani, Neuro-Fuzzy and Soft Computing, Prentice-Hall, New York, NY, USA, 1997.

[31] J. R. Castro, O. Castillo, P. Melin, and A. Rodriguez-Diaz, "A hybrid learning algorithm for interval type-2 fuzzy neural networks: the case of time series prediction," in Soft Computing For Hybrid Intelligent Systems, vol. 154, pp. 363-386, Springer, Berlin, Germany, 2008.

[32] J. R. Castro, O. Castillo, P. Melin, and A. Rodriguez-Diaz, "Hybrid learning algorithm for interval type-2 fuzzy neural networks," in Proceedings of Granular Computing, pp. 157-162, Silicon Valley, Calif, USA, 2007.

[33] F. Lin and P. Chou, "Adaptive control of two-axis motion control system using interval type-2 fuzzy neural network," IEEE Transactions on Industrial Electronics, vol. 56, no. 1, pp. 178-193, 2009.
[34] R. Ceylan, Y. Özbay, and B. Karlik, "Classification of ECG arrhythmias using type-2 fuzzy clustering neural network," in Proceedings of the 14th National Biomedical Engineering Meeting (BIYOMUT '09), pp. 1-4, May 2009.

[35] J. R. Castro, O. Castillo, P. Melin, and A. Rodríguez-Díaz, "A hybrid learning algorithm for a class of interval type-2 fuzzy neural networks," Information Sciences, vol. 179, no. 13, pp. 21752193, 2009.

[36] C. T. Scarborough and A. H. Stone, "Products of nearly compact spaces," Transactions of the American Mathematical Society, vol. 124, pp. 131-147, 1966.

[37] R. E. Edwards, Functional Analysis: Theory and Applications, Dover, 1995.

[38] W. Rudin, Principles of Mathematical Analysis, McGraw-Hill, New York, NY, USA, 1976.

[39] M. C. Mackey and L. Glass, "Oscillation and chaos in physiological control systems," Science, vol. 197, no. 4300, pp. 287-289, 1977.

[40] T. Hastie, R. Tibshirani, and J. Friedman, The Elements of Statistical Learning, Springer, 2001.

[41] S. Theodoridis and K. Koutroumbas, Pattern Recognition, Academic Press, 1999. 

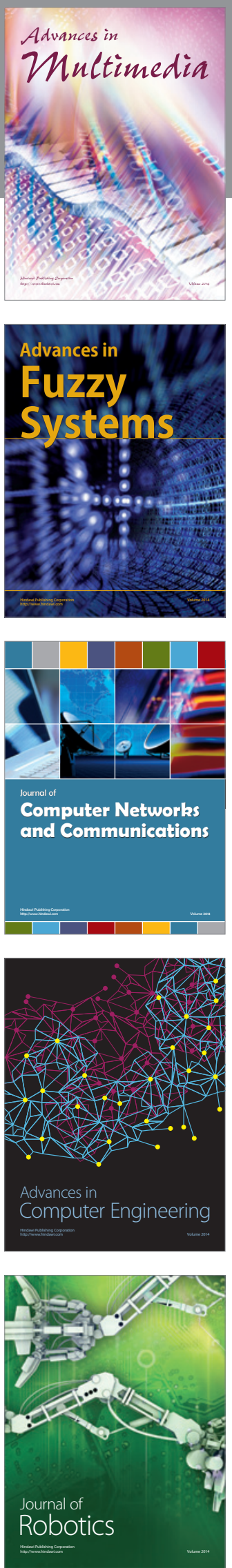

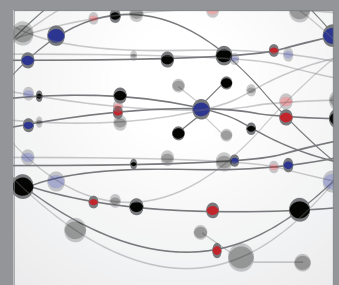

The Scientific World Journal
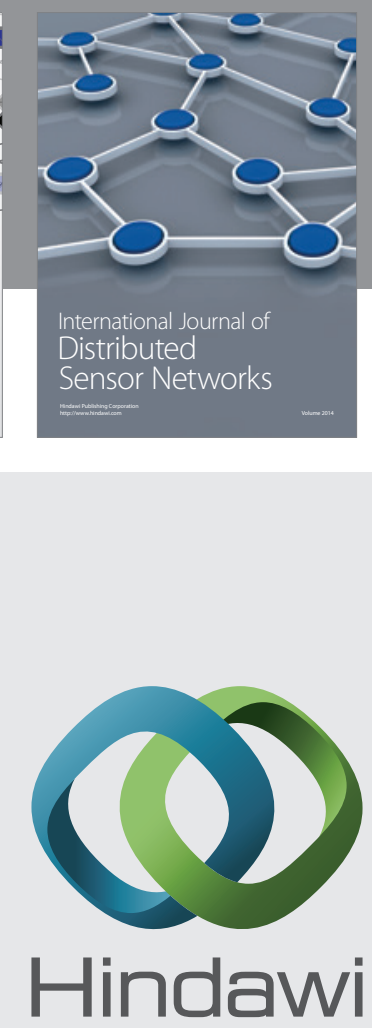

Submit your manuscripts at

http://www.hindawi.com
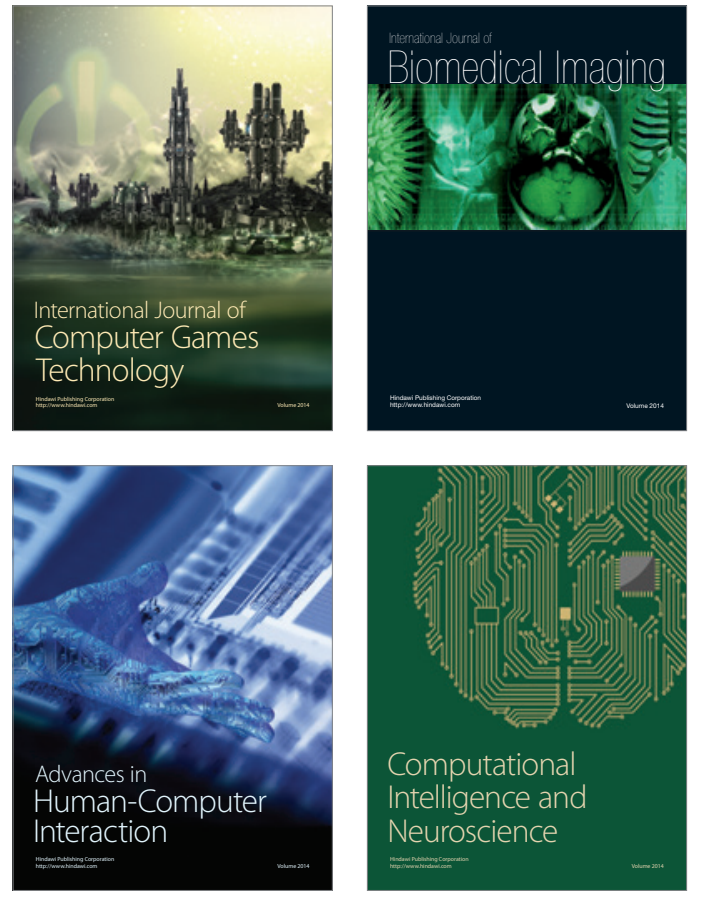
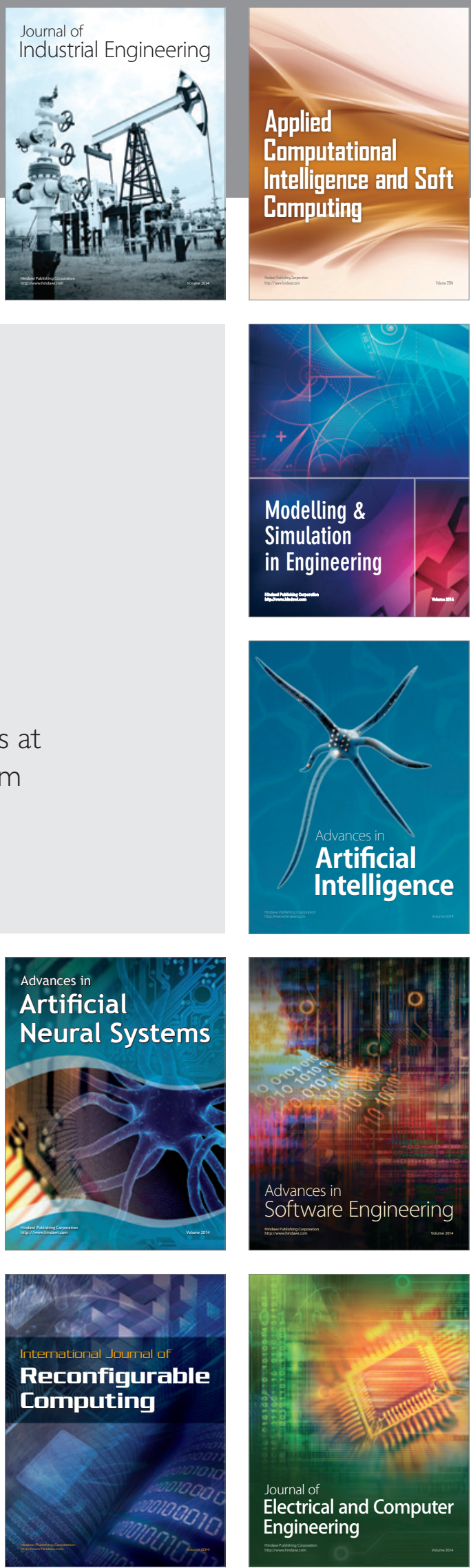